\title{
Boundary Value Problem for Nonlinear Implicit Generalized Hilfer-Type Fractional Differential Equations with Impulses
}

\author{
Abdelkrim Salim $\left(\mathbb{D},{ }^{1}\right.$ Mouffak Benchohra ${ }^{(D)}{ }^{1}$ Jamal Eddine Lazreg $\left(\mathbb{D},{ }^{1}\right.$ \\ and Gaston N'Guérékata iD ${ }^{2}$ \\ ${ }^{1}$ Laboratory of Mathematics, Djillali Liabes University of Sidi Bel Abbes, P.O. Box 89 Sidi Bel Abbes 22000, Algeria \\ ${ }^{2}$ NEERLab, Department of Mathematics, Morgan State University, 1700 E. Cold, Spring Lane, Baltimore M.D. 21252, USA \\ Correspondence should be addressed to Gaston N’Guérékata; nguerekata@aol.com
}

Received 5 January 2021; Revised 2 March 2021; Accepted 8 April 2021; Published 21 April 2021

Academic Editor: Nasser-Eddine Tatar

Copyright (c) 2021 Abdelkrim Salim et al. This is an open access article distributed under the Creative Commons Attribution License, which permits unrestricted use, distribution, and reproduction in any medium, provided the original work is properly cited.

This article deals with some existence, uniqueness, and Ulam-Hyers-Rassias stability results for a class of boundary value problem for nonlinear implicit fractional differential equations with impulses and generalized Hilfer Fractional derivative. The results are obtained using the Banach contraction principle and Krasnoselskii's and Schaefer's fixed-point theorems.

\section{Introduction}

Differential equations of fractional order have been recently proved to be a powerful tool to study many phenomena in various fields of science and engineering such as electrochemistry, finance, hydrology, electromagnetics, and viscoelasticity. There are numerous books and articles focused on linear and nonlinear initial and boundary value problems for fractional differential equations involving different kinds of fractional derivatives, see, for example, [1-6]. Impulsive fractional differential equations have been considered by many authors (see, for instance, [7-12]). Recent results involving different fractional derivatives can be found in [13-21] and the references therein.

Ulam was the first who raise the concept of stability of functional equations [22]. In 1941, Hyers [23] provided the first answer to Ulam's question. Thereafter, this type of stability is called the Ulam-Hyers stability. In 1978, Rassias [24] was able to make a remarkable generalization of UlamHyers stability of mappings by considering variables.

Considerable attention has been given to the study of the Ulam-Hyers and Ulam-Hyers-Rassias stability of all kinds of functional equations; one can see the monograph of Abbas et al. [3] and the paper by Rus [25] who discussed the
Ulam-Hyers stability for operational equations (see also [26-29]).

Recently, in [30], Harikrishnan et al. investigated existence theory and different kinds of stability in the sense of Ulam, for the following initial value problem with nonlinear generalized Hilfer-type fractional differential equation and impulses:

$$
\left\{\begin{array}{l}
\left(\rho D^{\alpha, \beta} u\right)(t)=f(t, u(t)), t \in \bar{I}:=I \backslash\left\{t_{1}, \cdots, t_{m}\right\}, I:=[0, b], \\
\left.\Delta^{\rho} \mathcal{J}^{1-\gamma} u(t)\right|_{t=t_{k}}=L_{k}\left(u\left(t_{k}^{-}\right)\right), k=1, \cdots, m, \\
\rho \mathcal{J}^{1-\gamma} u(0)=u_{0},
\end{array}\right.
$$

where ${ }^{\rho} D^{\alpha, \beta}$ and ${ }^{\rho} \mathscr{g}^{1-\gamma}$ are a generalized Hilfer fractional derivative of order $\alpha \in(0,1)$ and type $\beta \in[0,1]$ and generalized fractional integral of order $1-\gamma(\gamma=\alpha+\beta-\alpha \beta)$, respectively; $0=t_{0}<t_{1}<\cdots<\mathrm{t}_{m}<t_{m+1}=b<\infty, u\left(t_{k}^{+}\right)=\lim _{\varepsilon \rightarrow 0^{+}} u\left(t_{k}+\varepsilon\right)$, and $u\left(t_{k}^{-}\right)=\lim _{\varepsilon \rightarrow 0^{-}} u\left(t_{k}+\varepsilon\right)$ represent the right and left hand limits of $u(t)$ at $t=t_{k} ;\left.\Delta^{\rho} g^{1-\gamma} u(t)\right|_{t=t_{k}}=\rho g^{1-\gamma} u\left(t_{k}^{+}\right)-^{\rho} g^{1-\gamma} u\left(t_{k}^{-}\right) ; f$ 
$: I \times \mathbb{R} \rightarrow \mathbb{R}$ is a given function; and $L_{k}: \mathbb{R} \rightarrow \mathbb{R} ; k=1, \cdots, m$ are given continuous functions.

Motivated by the works mentioned above, in this paper, we establish existence and uniqueness results to the boundary value problem with nonlinear implicit generalized Hilfer-type fractional differential equation and impulses:

$$
\left({ }^{\rho} D_{t_{k}^{+}}^{\alpha, \beta} u\right)(t)=f\left(t, u(t),\left({ }^{\rho} D_{t_{k}^{+}}^{\alpha, \beta} u\right)(t)\right), t \in J_{k}, k=0, \cdots, m,
$$

$$
\begin{gathered}
\left(\rho \mathcal{J}_{t_{k}^{+}}^{1-\gamma} u\right)\left(t_{k}^{+}\right)=\left(\rho \mathcal{g}_{t_{k-1}^{+}}^{1-\gamma} u\right)\left(t_{k}^{-}\right)+L_{k}\left(u\left(t_{k}^{-}\right)\right), k=1, \cdots, m, \\
c_{1}\left(\rho \mathcal{g}_{a^{+}}^{1-\gamma} u\right)\left(a^{+}\right)+c_{2}\left(\rho \mathcal{F}_{t_{m}^{+}}^{1-\gamma} u\right)(b)=c_{3}
\end{gathered}
$$

where ${ }^{\rho} D_{t_{k}^{+}}^{\alpha, \beta}, \rho \mathcal{J}_{t_{k}^{+}}^{1-\gamma}$ are a generalized Hilfer fractional derivative of order $\alpha \in(0,1)$ and type $\beta \in[0,1]$ and generalized fractional integral of order $1-\gamma(\gamma=\alpha+\beta-\alpha \beta)$, respectively; $c_{1}, c_{2}, c_{3}$ are real with $c_{1}+c_{2} \neq 0 ; J_{k}:=\left(t_{k}, t_{k+1}\right], k=0, \cdots, m$; $a=t_{0}<t_{1}<\cdots<t_{m}<t_{m+1}=b<\infty ; u\left(t_{k}^{+}\right)=\lim _{\epsilon \rightarrow 0^{+}} u\left(t_{k}+\epsilon\right)$ and $\lim _{\epsilon \rightarrow 0^{-}} u\left(t_{k}+\epsilon\right)$ represent the right and left hand limits of $u(t)$ at $t=t_{k} ; f:(a, b] \times \mathbb{R} \times \mathbb{R} \rightarrow \mathbb{R}$ is a given function; and $L_{k}: \mathbb{R} \rightarrow \mathbb{R}, k=1, \cdots, m$, are given continuous functions.

The present paper is organized as follows. In Section 2 , some notations are introduced and we recall some preliminaries about generalized Hilfer fractional derivative and auxiliary results. In Section 3, three results for problems (2)-(4) are presented which are based on the Banach contraction principle and Krasnoselskii's and Schaefer's fixed-point theorems. In Section 4, we discuss the UlamHyers-Rassias stability for problems (2)-(4). Finally, we give examples to illustrate the applicability of our main results.

\section{Preliminaries}

In this section, we introduce notations, definitions, and preliminary facts which are used throughout this paper. Let 0 $<a<b, J=[a, b]$. By $C$, we denote the Banach space of all continuous functions from $J$ into $\mathbb{R}$ with the norm

$$
\|u\|_{\infty}=\sup \{|u(t)|: t \in J\}
$$

We consider the weighted spaces of continuous functions

$$
\begin{gathered}
C_{\gamma, \rho}(J)=\left\{u:(a, b] \rightarrow \mathbb{R}:\left(\frac{t^{\rho}-a^{\rho}}{\rho}\right)^{1-\gamma} u(t) \in C(J, \mathbb{R})\right\}, 0 \leq \gamma<1, \\
C_{\gamma, \rho}^{n}(J)=\left\{u \in C^{n-1}(J): u^{(n)} \in C_{\gamma, \rho}(J)\right\}, n \in \mathbb{N}, C_{\gamma, \rho}^{0}(J)=C_{\gamma, \rho}(J),
\end{gathered}
$$

with the norms

$$
\begin{aligned}
& \|u\|_{C_{\gamma, \rho}}=\sup _{t \in J}\left|\left(\frac{t^{\rho}-a^{\rho}}{\rho}\right)^{1-\gamma} u(t)\right|, \\
& \|u\|_{C_{\gamma, \rho}^{n}}=\sum_{k=0}^{n-1}\left\|u^{(k)}\right\|_{\infty}+\left\|u^{(n)}\right\|_{C_{\gamma, \rho}} .
\end{aligned}
$$

Consider the Banach space $P C(J)=\{u:(a, b] \rightarrow \mathbb{R}: u$ $(t) \in C(J k), k=0, \cdots, m$, and there exist $u\left(t_{k}^{-}\right)$and $\left(\rho \mathcal{F}_{t_{k}^{+}}^{1-\gamma}\right.$ $u)\left(t_{k}^{+}\right), k=0, \cdots, m$, with $\left.u\left(t_{k}^{-}\right)=u\left(t_{k}\right)\right\}, 0 \leq \gamma<1$.

Also, we consider the weighted space

$$
\begin{aligned}
& P C_{\gamma, \rho}(J)=\left\{u(t) ;\left(\frac{t^{\rho}-t_{k}^{\rho}}{\rho}\right)^{1-\gamma} u(t) \in P C(J)\right\}, 0 \geq \gamma<1, \\
& P C_{\gamma, \rho}^{n}(J)=\left\{u \in P C^{n-1}: u^{(n)} \in P C_{\gamma, \rho}(J)\right\}, n \in \mathbb{N}, \\
& P C_{\gamma, \rho}^{0}(J)=P C_{\gamma, \rho}(J),
\end{aligned}
$$

with the norm

$$
\|u\|_{P C_{\gamma, \rho}}=\sup _{t \in J_{k}}\left|\left(\frac{t^{\rho}-t_{k}^{\rho}}{\rho}\right)^{1-\gamma} u(t)\right|, k=0, \cdots, m .
$$

Consider the space $X_{c}^{p}(a, b)(c \in \mathbb{R}, 1 \leq p \leq \infty)$ of those complex-valued Lebesgue measurable functions $f$ on $[a, b]$ for which $\|f\|_{X_{c}^{p}}<\infty$, where the norm is defined by

$$
\|f\|_{X_{c}^{p}}=\left(\int_{a}^{b}\left|t^{c} f(t)\right|^{p} \frac{d t}{t}\right)^{1 / p}(1 \leq p<\infty, c \in \mathbb{R}) .
$$

In particular, when $c=1 / p$, the space $X_{c}^{p}(a, b)$ coincides with the $L_{p}(a, b)$ space: $X_{1 / p}^{p}(a, b)=L_{p}(a, b)$.

Definition 1 [31]. Generalized Hilfer fractional integral.

Let $\alpha \in \mathbb{R}_{+}, c \in \mathbb{R}$, and $g \in X_{c}^{p}(a, b)$. A generalized Hilfer fractional integral of order $\alpha$ is defined by

$$
\left(\rho \mathcal{g}_{a^{+}}^{\alpha}+g\right)(t)=\int_{a}^{t} s^{\rho-1}\left(\frac{t^{\rho}-s^{\rho}}{\rho}\right)^{\alpha-1} \frac{g(s)}{\Gamma(\alpha)} d s, t>a, \rho>0,
$$

where $\Gamma(\cdot)$ is the Euler gamma function defined by $\Gamma(\alpha)=$ $\int_{0}^{\infty} t^{\alpha-1} e^{-t} d t, \alpha>0$.

Definition 2 [31]. Generalized Hilfer fractional derivative. 
Let $\alpha \in \mathbb{R}_{+} \backslash \mathbb{N}$ and $\rho>0$. A generalized Hilfer fractional derivative ${ }^{\rho} D_{a^{+}}^{\alpha}$ of order $\alpha$ is defined by

$$
\begin{aligned}
\left({ }^{\rho} D_{a^{+}}^{\alpha}+g\right)(t)= & \delta_{\rho}^{n}\left({ }^{\rho} g_{a^{+}}^{n-\alpha} g\right)(t) \\
= & \left(t^{1}-\rho \frac{d}{d t}\right) \int_{a}^{n} s^{\rho-1}\left(\frac{t^{\rho}-s^{\rho}}{\rho}\right)^{n-\alpha-1} \\
& \cdot \frac{g(s)}{\Gamma(n-\alpha)} d s, t>a, \rho>0,
\end{aligned}
$$

where $n=[\alpha]+1$ and $\delta_{\rho}^{n}=\left(t^{1-\rho}(d / d t)\right)^{n}$.

Theorem 3 [31]. Let $\alpha>0, \beta>0,1 \leq p \leq \infty$, and $0<a<b$ $<\infty$. Then, for $g \in X_{c}^{p}(a, b)$, the semigroup property is valid, i.e.,

$$
\left(\rho \mathscr{J}_{a^{+}}^{\alpha}{ }^{\rho} \mathscr{J}_{a^{+}}^{\beta} g\right)(t)=\left(\rho \mathscr{J}_{a^{+}}^{\alpha+\beta} g\right)(t)
$$

Lemma $4[31,32]$. Let $\alpha>0$, and $0 \leq \gamma<1$. Then, $\rho^{\rho} \mathcal{F}_{a^{+}}^{\alpha}$ is bounded from $P C_{\gamma, \rho}(J)$ into $P C_{\gamma, \rho}(J)$

Lemma 5 [32]. Let $0<a<b<\infty, \alpha>0,0 \leq \gamma<1$, and $u \in P$ $C_{\gamma, \rho}(J)$. If $\alpha>1-\gamma$, then ${ }^{\rho} \mathcal{J}_{a^{+}}^{\alpha}$ is continuous on $J$ and

$$
\left({ }^{\rho} \mathscr{F}_{a^{+}}^{\alpha} u\right)(a)=\lim _{t \rightarrow a^{+}}\left({ }^{\rho} \mathscr{F}_{a^{+}}^{\alpha} u\right)(t)=0
$$

Lemma 6 [33]. Let $t>a$. Then, for $\alpha \geq 0$ and $\beta>0$, we have

$$
\begin{aligned}
& {\left[\rho \mathcal{J}_{a^{+}}^{\alpha}\left(\frac{s^{\rho}-a^{\rho}}{\rho}\right)^{\beta-1}\right](t)=\frac{\Gamma(\beta)}{\Gamma(\alpha+\beta)}\left(\frac{t^{\rho}-a^{\rho}}{\rho}\right)^{\alpha+\beta-1},} \\
& {\left[{ }^{\rho} D_{a^{+}}^{\alpha}\left(\frac{s^{\rho}-a^{\rho}}{\rho}\right)^{\alpha-1}\right](t)=0, \quad 0<\alpha<1 .}
\end{aligned}
$$

Lemma 7 [32]. Let $\alpha>0,0 \leq \gamma<1$, and $g \in P C_{\gamma}[a, b]$. Then,

$$
\left({ }^{\rho} D_{a^{+}}^{\alpha}{ }^{\rho} \mathscr{F}_{a^{+}}^{\alpha} g\right)(t)=g(t), \text { for all } t \in(a, b] .
$$

Lemma 8 [32]. Let $0<\alpha<1,0 \leq \gamma<1$. If $g \in P C_{\gamma, \rho}[a, b]$ and $\rho \mathcal{F}_{a^{+}}^{1-\alpha} g \in P C_{\gamma, \rho}^{1}[a, b]$, then

$\left(\rho \mathcal{F}_{a^{+}}^{\alpha} \rho D_{a^{+}}^{\alpha} g\right)(t)=g(t)-\frac{\left(\rho \mathcal{F}_{a^{+}}^{1-\alpha} g\right)(a)}{\Gamma(\alpha)}\left(\frac{t^{\rho}-a^{\rho}}{\rho}\right)^{\alpha-1}$, for all $t \in(a, b]$.

Definition 9 [32]. Let order $\alpha$ and type $\beta$ satisfy $n-1<\alpha<n$ and $0 \leq \beta \leq 1$, with $n \in \mathbb{N}$. A generalized Hilfer fractional derivative to $t$, with $\rho>0$ of a function $g \in P C_{\gamma, \rho}[a, b]$, is defined by

$$
\begin{aligned}
\left({ }^{\rho} D_{a^{+}}^{\alpha, \beta} g\right)(t) & =\left(\rho \mathscr{J}_{a^{+}}^{\beta(n-\alpha)}\left(t^{\rho-1} \frac{d}{d t}\right)^{n \rho} \mathscr{J}_{a^{+}}^{(1-\beta)(n-\alpha)} g\right)(t) \\
& =\left({ }^{\rho} \mathscr{J}_{a^{+}}^{\beta(n-\alpha)} \delta_{\rho}^{n \rho} \mathscr{J}_{a^{+}}^{(1-\beta)(n-\alpha)} g\right)(t) .
\end{aligned}
$$

In this paper, we consider the case $n=1$ only, because 0 $<\alpha<1$.

Property 10 [32]. The operator ${ }^{\rho} D_{a^{+}}^{\alpha, \beta}$ can be written as

$$
{ }^{\rho} D_{a^{+}}^{\alpha, \beta}={ }^{\rho} \mathcal{J}_{a^{+}}^{\beta(1-\alpha)} \delta_{\rho}^{\rho} \mathcal{F}_{a^{+}}^{1-\gamma}={ }^{\rho} \mathcal{F}_{a^{+}}^{\beta(1-\alpha)}{ }^{\rho} D_{a^{+}}^{\gamma}, \gamma=\alpha+\beta-\alpha \beta .
$$

Property 11 [32]. The fractional derivative ${ }^{\rho} D_{a^{+}}^{\alpha, \beta}$ is an interpolator of the following fractional derivatives: Hilfer $(\rho \rightarrow 1)$, Hilfer-Hadamard $\left(\rho \rightarrow 0^{+}\right)$, generalized $(\beta=0)$, Caputo-type $(\beta=1)$, Riemann-Liouville $(\beta=0, \rho \rightarrow 1)$, Hadamard $(\beta=0$, $\left.\rho \rightarrow 0^{+}\right)$, Caputo $(\beta=1, \rho \rightarrow 1)$, Caputo-Hadamard $(\beta=1$, $\left.\rho \rightarrow 0^{+}\right)$, Liouville $(\beta=0, \rho \rightarrow 1, a=0)$, and Weyl $(\beta=0, \rho$ $\rightarrow 1, a=-\infty)$.

Consider the following parameters $\alpha, \beta, \gamma$ satisfying

$$
\gamma=\alpha+\beta-\alpha \beta, \quad 0<\alpha, \beta, \gamma<1
$$

We define the spaces

$$
\begin{aligned}
& P C_{\gamma, \rho}^{\alpha, \beta}(J)=\left\{u \in P C_{\gamma, \rho}(J),{ }^{\rho} D_{t_{k}^{+}}^{\alpha, \beta} u \in P C_{\gamma, \rho}(J)\right\}, \\
& P C_{\gamma, \rho}^{\gamma}(J)=\left\{u \in P C_{\gamma, \rho}(J),{ }^{\rho} D_{t_{k}^{+}}^{\gamma} u \in P C_{\gamma, \rho}(J)\right\},
\end{aligned}
$$

where $k=0, \cdots, m$.

Since ${ }^{\rho} D_{t_{k}^{+}}^{\alpha, \beta} u=\rho \mathscr{J}_{t_{k}^{+}}^{\gamma(1-\alpha)^{\rho}} D_{t_{k}^{+}}^{\gamma} u$, it follows from Lemma 4 that

$$
P C_{\gamma, \rho}^{\gamma}(J) \subset P C_{\gamma, \rho}^{\alpha, \beta}(J) \subset P C_{\gamma, \rho}(J)
$$

Lemma 12 [32]. Let $0<\alpha<1,0 \leq \beta \leq 1$, and $\gamma=\alpha+\beta-\alpha \beta$. If $u \in P C_{\gamma, \rho}^{\gamma}(J)$, then

$$
\begin{aligned}
& \rho \mathscr{J}_{a^{+}}^{\gamma \rho} D_{a^{+}}^{\gamma} u={ }^{\rho} \mathscr{J}_{a^{+}}^{\alpha}{ }^{\rho} D_{a^{+}}^{\alpha, \beta} u, \\
& { }^{\rho} D_{a^{+}}^{\gamma \rho} \mathscr{J}_{a^{+}}^{\alpha} u={ }^{\rho} D_{a^{+}}^{\beta(1-\alpha)} u .
\end{aligned}
$$

Lemma 13 (Theorem 25 [32]). Let $f:(a, b] \times \mathbb{R} \rightarrow \mathbb{R}$ be $a$ function such that $f\left(\cdot, u(\cdot),{ }^{\rho} D_{t_{k}^{+}}^{\alpha, \beta} u(\cdot)\right) \in C_{\gamma, \rho}(J)$, for any $u \in$ 
$P C_{\gamma, \rho}(J)$. Then, $u \in P C_{\gamma, \rho}^{\gamma}(J)$ is a solution of the differential equation:

$$
\begin{aligned}
& \left({ }^{\rho} D_{t_{k}^{+}}^{\alpha, \beta} u\right)(t) \\
& \quad=f\left(t, u(t),{ }^{\rho} D_{t_{k}^{+}}^{\alpha, \beta} u(t)\right), \text { for each } t \in J_{k}, k=0, \cdots, m, 0<\alpha<1,0 \leq \beta \leq 1,
\end{aligned}
$$

if and only if $u$ satisfies the following Volterra integral equation:

$$
\begin{aligned}
u(t)= & \frac{\left(\rho \mathcal{F}_{t_{k}^{+}}^{1-\gamma} u\right)\left(t_{k}^{+}\right)}{\Gamma(\gamma)}\left(\frac{t^{\rho}-t_{k}^{\rho}}{\rho}\right)^{\gamma-1}+\frac{1}{\Gamma(\alpha)} \int_{t_{k}}^{t} \\
& \cdot\left(\frac{t^{\rho}-s^{\rho}}{\rho}\right)^{\alpha-1} s^{\rho-1} f\left(s, u(s),{ }^{\rho} D_{t_{k}^{+}}^{\alpha, \beta} u(s)\right) d s
\end{aligned}
$$

where $\gamma=\alpha+\beta-\alpha \beta$.

Theorem 14 ([34]) (Banach's fixed-point theorem). Let $D$ be a nonempty closed subset of a Banach space E; then, any contraction mapping $N$ of $D$ into itself has a unique fixed point.

Theorem 15 ([34]) (Schaefer's fixed-point theorem). Let $E$ be a Banach space and $N: E \rightarrow E$ be a completely continuous operator. If the set

$$
D=\{u \in E: u=\lambda N u, \quad \text { for some } \lambda \in(0,1)\},
$$

is bounded, then $N$ has a fixed point.

Theorem 16 ([34]) (Krasnoselskii's fixed-point theorem). Let $D$ be a closed, convex, and nonempty subset of a Banach space $E$ and the operators $A, B$ such that

(1) $A x+B y \in D$, for all $x, y \in D$

(2) A is compact and continuous

(3) $B$ is a contraction mapping

Then, there exists $z \in D$ such that $z=A z+B z$.

Now, we consider the Ulam stability for problems $((2))-((4))$ that will be used in Section 4. Let $u \in P C_{\gamma, \rho}$ $(J), \epsilon>0, \tau>0$, and $\vartheta:(a, b] \longrightarrow[0, \infty)$ be a continuous function.

We consider the following inequality:

$$
\left\{\begin{array}{l}
\left|\left({ }^{\rho} D_{t_{k}^{+}}^{\alpha, \beta} u\right)(t)-f\left(t, u(t),\left({ }^{\rho} D_{t_{k}^{+}}^{\alpha, \beta} u\right)(t)\right)\right| \leq \epsilon \vartheta(t), t \in J_{k}, k=0, \cdots, m, \\
\left|\left(\rho^{\rho} \mathcal{F}_{t_{k}^{+}}^{1-\gamma} u\right)\left(t_{k}^{+}\right)-\left(\rho^{\rho} \mathcal{F}_{t_{k-1}^{+}}^{1-\gamma} u\right)\left(t_{k}^{-}\right)-L_{k}\left(u\left(t_{k}^{-}\right)\right)\right| \leq \epsilon \tau, k=1, \cdots, m .
\end{array}\right.
$$

Definition 17 ([35]). Problems ((2))-((4)) are Ulam-HyersRassias (U-H-R) stable with respect to $(\vartheta, \tau)$ if there exists a real number $a_{f, m, \vartheta}>0$ such that for each $\epsilon>0$ and for each solution $u \in P C_{\gamma, \rho}(J)$ of inequality (27), there exists a solution $w \in P C_{\gamma, \rho}(J)$ of (1)-(3) with

$$
|u(t)-w(t)| \leq \epsilon a_{f, m, \vartheta}(\vartheta(t)+\tau), \quad t \in(a, b] .
$$

Remark 18 ([35]). A function $u \in P C_{\gamma, \rho}(J)$ is a solution of inequality (27) if and only if there exist $\sigma \in P C_{\gamma, \rho}(J)$ and a sequence $\sigma_{k}, k=0, \cdots, m$, such that

(1) $|\sigma(t)| \leq \epsilon \vartheta(t)$ and $\left|\sigma_{k}\right| \epsilon \tau, t \in J_{k}, k=1, \cdots, m$

(2) $\left({ }^{\rho} D_{t_{k}^{+}}^{\alpha, \beta} u\right)(t)=f\left(t, u(t),\left({ }^{\rho} D_{t_{k}^{+}}^{\alpha, \beta} u\right)(t)\right)+\sigma(t), t \in J_{k}, k=$ $0, \cdots, m$

(3) $\left(\rho \mathcal{F}_{t_{k}^{+}}^{1-\gamma} u\right)\left(t_{k}^{+}\right)=\left(\rho \mathcal{F}_{t_{k-1}^{+}}^{1-\gamma} u\right)\left(t_{k}^{-}\right)+L_{k}\left(u\left(t_{k}^{-}\right)\right)+\sigma_{k}, k=1$, $\cdots, m$

\section{Existence of Solutions}

We consider the following linear fractional differential equation:

$$
\left({ }^{\rho} D_{t_{k}^{+}}^{\alpha, \beta} u\right)(t)=\psi(t), t \in J_{k}, k=0, \cdots, m
$$

where $0<\alpha<1,0 \leq \beta \leq 1$, and $\rho>0$, with the conditions

$$
\left(\rho \mathscr{J}_{t_{k}^{+}}^{1-\gamma} u\right)\left(t_{k}^{+}\right)=\left(\rho \mathcal{F}_{t_{k-1}^{+}}^{1-\gamma} u\right)\left(t_{k}^{-}\right)+L_{k}\left(u\left(t_{k}^{-}\right)\right), \quad k=1, \cdots, m,
$$

and

$$
c_{1}\left(\rho \mathscr{J}_{a^{+}}^{1-\gamma} u\right)\left(a^{+}\right)+c_{2}\left(\rho \mathscr{J}_{t_{m}^{+}}^{1-\gamma} u\right)(b)=c_{3},
$$

where $\gamma=\alpha+\beta-\alpha \beta$ and $c_{1}, c_{2}, c_{3} \in \mathbb{R}$ with

$$
\begin{aligned}
c_{1}+c_{2} & \neq 0, \\
\xi_{1} & =\frac{c_{2}}{c_{1}+c_{2}}, \\
\xi_{2} & =\frac{c_{3}}{c_{1}+c_{2}},
\end{aligned}
$$

and

$$
p^{*}=\sup \left\{\left(\frac{t_{k}^{\rho}-t_{k-1}^{\rho}}{\rho}\right)^{\gamma-1}: k=1, \cdots, m\right\} \text {. }
$$


The following theorem shows that problems ((29))-((31)) have a unique solution given by

$u(t)=\left\{\begin{array}{l}\frac{1}{\Gamma(\gamma)}\left(\frac{t^{\rho}-a^{\rho}}{\rho}\right)^{\gamma-1}\left[\xi_{2}-\xi_{1} \sum_{i=1}^{m} L_{i}\left(u\left(t_{i}^{-}\right)\right)-\xi_{1} \sum_{i=1}^{m}\left(\rho^{\rho} \mathcal{F}_{\left(t_{i-1}\right)}^{1-\gamma+\alpha}\right)\left(t_{i}\right)-\xi_{1}\left({ }^{\rho} \mathcal{F}_{\left(t_{m}\right)}^{1-\gamma+\alpha} \psi\right)(b)\right]+\frac{1}{\Gamma(\alpha)} \int_{a}^{t}\left(\frac{t^{\rho}-s^{\rho}}{\rho}\right)^{\alpha-1} s^{\rho-1} \psi(s) d s \text { if } t \in J_{0}, \\ \frac{1}{\Gamma(\gamma)}\left(\frac{t^{\rho}-t_{k}^{\rho}}{\rho}\right)^{\gamma-1}\left[\xi_{2}-\xi_{1} \sum_{i=1}^{m} L_{i}\left(u\left(t_{i}^{-}\right)\right)-\xi_{1} \sum_{i=1}^{m}\left(\rho \mathcal{F}_{\left(t_{i-1}\right)}^{1-\gamma+\alpha} \psi\right)\left(t_{i}\right)-\xi_{1}\left({ }^{\rho} \mathcal{F}_{\left(t_{m}\right)}^{1-\gamma+\alpha} \psi\right)(b)+\sum_{i=1}^{k} L_{i}\left(u\left(t_{i}^{-}\right)\right)+\sum_{i=1}^{k} L_{i}\left(\rho \mathcal{F}_{\left(t_{i-1}\right)}^{1-\gamma+\alpha} \psi\right)\left(t_{i}\right)\right]+\left({ }^{\rho} \mathcal{F}_{\left(t_{k}\right)}^{\alpha} \psi\right)(t) \text { if } t \in J_{k}, k=1, \cdots, m .\end{array}\right.$

Theorem 19. Let $\gamma=\alpha+\beta-\alpha \beta$, where $0<\alpha<1$ and $0 \leq \beta$ $\leq 1$. If $\psi:(a, b] \rightarrow \mathbb{R}$ is a function such that $\psi(\cdot) \in C_{\gamma, \rho}(J)$, then $u \in P C_{\gamma, \rho}^{\gamma}(J)$ satisfies problems ((29))-((31)) if and only if it satisfies (34).

Proof. Assume $u$ satisfies (29)-(31). If $t \in J_{0}$, then

$$
\left({ }^{\rho} D_{a^{+}}^{\alpha, \beta} u\right)(t)=\psi(t)
$$

Lemma 20. implies that

$u(t)=\frac{\left(\rho \mathcal{F}_{a^{+}}^{1-\gamma} u\right)\left(a^{+}\right)}{\Gamma(\gamma)}\left(\frac{t^{\rho}-a^{\rho}}{\rho}\right)^{\gamma-1}+\frac{1}{\Gamma(\alpha)} \int_{a}^{t}\left(\frac{t^{\rho}-s^{\rho}}{\rho}\right)^{\alpha-1} s^{\rho-1} \psi(s) d s$.

If $t \in J_{1}$, then Lemma 13 implies

$$
\begin{aligned}
u(t)= & \frac{\left(\rho \mathcal{F}_{t_{1}^{+}}^{1-\gamma} u\right)\left(t_{1}^{+}\right)}{\Gamma(\gamma)}\left(\frac{t^{\rho}-t_{1}^{\rho}}{\rho}\right)^{\gamma-1}+\frac{1}{\Gamma(\alpha)} \int_{t_{1}}^{t} \\
& \cdot\left(\frac{s^{\rho}-s^{\rho}}{\rho}\right)^{\alpha-1} s^{\rho-1} \psi(s) d s \\
= & \frac{\left(\rho \mathcal{F}_{a^{+}}^{1-\gamma} u\right)\left(t_{1}^{-}\right)+L_{1}\left(u\left(t_{1}^{-}\right)\right)}{\Gamma(\gamma)}\left(\frac{t^{\rho}-t_{1}^{\rho}}{\rho}\right)^{\gamma-1}+\left(\rho \mathscr{F}_{t_{1}^{+}}^{\alpha} \psi\right)(t) \\
= & \frac{\left(t^{\rho}-t_{1}^{\rho}\right)^{\gamma-1}}{\Gamma(\gamma) \rho^{\gamma-1}}\left[\left(\rho \mathscr{F}_{a^{+}}^{1-\gamma} u\right)\left(a^{+}\right)+L_{1}\left(u\left(t_{1}^{-}\right)\right)\right. \\
& \left.+\left(\rho \mathscr{F}_{a^{+}}^{1-\gamma+\alpha} \psi\right)\left(t_{i}\right)\right]+\left(\rho_{t_{1}}^{\alpha} \psi\right)(t) .
\end{aligned}
$$

If $t \in J_{2}$, then Lemma 13 implies

$$
\begin{aligned}
u(t)= & \frac{\left(\rho \mathcal{F}_{t_{2}^{+}}^{1-\gamma} u\right)\left(t_{2}^{+}\right)}{\Gamma(\gamma)}\left(\frac{t^{\rho}-t_{2}^{\rho}}{\rho}\right)^{\gamma-1}+\frac{1}{\Gamma(\alpha)} \int_{t_{2}}^{t} \\
& \cdot\left(\frac{t^{\rho}-s^{\rho}}{\rho}\right)^{\alpha-1} s^{\rho-1} \psi(s) d s \\
= & \frac{\left(\rho \mathcal{F}_{t_{1}^{+}}^{1-\gamma} u\right)\left(t_{2}^{-}\right) L_{2}\left(u\left(t_{2}^{-}\right)\right)}{\Gamma(\gamma)}\left(\frac{t^{\rho}-t_{2}^{\rho}}{\rho}\right)^{\gamma-1}+\left(\rho \mathcal{F}_{t_{2}^{+}}^{\alpha} \psi\right)(t) \\
= & \frac{1}{\Gamma(\gamma)}\left(\frac{t^{\rho}-t_{2}^{\rho}}{\rho}\right)^{\gamma-1}\left[\left(\rho \mathcal{F}_{a^{+}}^{1-\gamma} u\right)\left(a^{+}\right)+L_{1}\left(u\left(t_{1}^{-}\right)\right)+L_{2}\left(u\left(t_{2}^{-}\right)\right)\right. \\
& \left.+\left(\rho_{\mathcal{F}_{a^{+}}^{1-\gamma+\alpha}} \psi\right)\left(t_{1}\right)+\left(\rho \mathcal{F}_{t_{1}^{+}}^{1-\gamma+\alpha} \psi\right)\left(t_{2}\right)\right]+\left(\rho \mathcal{F}_{t_{2}^{+}}^{\alpha} \psi\right)(t) .
\end{aligned}
$$

Repeating the process in this way, the solution $u(t)$ for $t$ $\in J_{k}, k=1, \cdots, m$, can be written as

$$
\begin{aligned}
u(t)= & \frac{1}{\Gamma(\gamma)}\left(\frac{t^{\rho}-t_{k}^{\rho}}{\rho}\right)^{\gamma-1}\left[\left(\rho \mathcal{F}_{a^{+}}^{1-\gamma} u\right)\left(a^{+}\right)+\sum_{i=1}^{k} L_{i}\left(u\left(t_{i}^{-}\right)\right)\right. \\
& \left.+\sum_{i=1}^{k}\left(\rho \mathcal{F}_{\left(t_{i}-1\right)^{+}}^{1-\gamma+\alpha}\right)\left(t_{i}\right)\right]+\left(\rho \mathcal{F}_{t_{k}^{+}}^{\alpha} \psi\right)(t) .
\end{aligned}
$$

Applying ${ }^{\rho} \mathcal{F}_{t_{m}^{+}}^{1-\gamma}$ on both sides of (39), using Lemma 6 and taking $t=b$, we obtain

$$
\begin{aligned}
\left(\rho \mathcal{F}_{t_{m}^{+}}^{1-\gamma} u\right)(b)= & \left(\rho \mathcal{F}_{a^{+}}^{1-\gamma} u\right)\left(a^{+}\right)+\sum_{i=1}^{m} L_{i}\left(u\left(t_{i}^{-}\right)\right) \\
& +\sum_{i=1}^{m}\left(\rho \mathscr{F}_{\left(t_{i-1}\right)}^{1-\gamma+\alpha} \psi\right)\left(t_{i}\right)+\left(\rho \mathscr{F}_{\left(t_{m}\right)^{+}}^{1-\gamma+\alpha} \psi\right)(b) .
\end{aligned}
$$


Multiplying both sides of (40) by $c_{2}$ and using condition (31), we obtain

$$
\begin{aligned}
c_{3}-c_{1}\left(\rho \mathscr{J}_{a^{+}}^{1-\gamma} u\right)\left(a^{+}\right)= & c_{2}\left(\rho \mathcal{F}_{a^{+}}^{1-\gamma} u\right)\left(a^{+}\right)+c_{2} \sum_{i=1}^{m} L_{i}\left(u\left(t_{i}^{-}\right)\right) \\
& +c_{2} \sum_{i=1}^{m}\left(\rho \mathscr{J}_{\left(t_{i-1}\right)}^{1-\gamma+\alpha} \psi\right)\left(t_{i}\right) \\
& +c_{2}\left(\rho \mathscr{J}_{\left(t_{m}\right)^{+}}^{1-\gamma+\alpha} \psi\right)(b),
\end{aligned}
$$

which implies that

$$
\begin{aligned}
\left(\rho \mathscr{J}_{a^{+}}^{1-\gamma} u\right)\left(a^{+}\right)= & \xi_{2}-\xi_{1} \sum_{i=1}^{m} L_{i}\left(u\left(t_{i}^{-}\right)\right)-\xi_{1} \sum_{i=1}^{m}\left(\rho \mathcal{F}_{\left(t_{i-1}\right)}^{1-\gamma+\alpha} \psi\right)\left(t_{i}\right) \\
& -\xi_{1}\left(\rho \mathcal{J}_{\left(t_{m}\right)^{+}}^{1-\gamma+\alpha} \psi\right)(b) .
\end{aligned}
$$

Substituting (42) into (39) and (36), we obtain (34).

Reciprocally, applying ${ }^{\rho} \mathscr{J}_{t_{k}^{+}}^{1-\gamma}$ on both sides of (34) and using Lemma 6 and Theorem 3, we get

$\left(\rho \mathcal{F}_{t_{k}^{+}}^{1-\gamma} u\right)(t)=\left\{\begin{array}{l}\xi_{2}-\xi_{1} \sum_{i=1}^{m} L_{i}\left(u\left(t_{i}^{-}\right)\right)-\xi_{1} \sum_{i=1}^{m}\left(\rho \mathcal{F}_{\left(t_{i-1}\right)^{+}}^{1-\gamma+\alpha}\right)\left(t_{i}\right)-\xi_{1}\left(\rho \mathcal{F}_{\left(t_{m}\right)^{+}}^{1-\gamma+\alpha} \psi\right)(b)+\left(\rho \mathcal{F}_{a^{+}}^{1-\gamma+\alpha} \psi\right)(t) \quad \text { if } t \in J_{0}, \\ \xi_{2}-\xi_{1} \sum_{i=1}^{m} L_{i}\left(u\left(t_{i}^{-}\right)\right)-\xi_{1} \sum_{i=1}^{m}\left(\rho \mathcal{F}_{\left(t_{i-1}\right)^{+}}^{1-\gamma+\alpha}\right)\left(t_{i}\right)-\xi_{1}\left(\rho \mathcal{F}_{t_{m}^{+}}^{1-\gamma+\alpha} \psi\right)(b)+\sum_{i=1}^{k} L_{i}\left(u\left(t_{i}^{-}\right)\right)+\sum_{i=1}^{k}\left(\rho \mathcal{F}_{\left(t_{i-1}\right)^{+}}^{1-\gamma+\alpha}\right)\left(t_{i}\right)+\left(\rho \mathscr{F}_{t_{k}^{+}}^{1-\gamma+\alpha} \psi\right)(t) \quad \text { if } t \in J_{k}, k=1, \cdots, m .\end{array}\right.$

Next, taking the limit $t \rightarrow a^{+}$of (43) and using Lemma 5, with $1-\gamma<1-\gamma+\alpha$, we obtain

$$
\begin{aligned}
\left(\rho \mathcal{F}_{a^{+}}^{1-\gamma} u\right)\left(a^{+}\right)= & \xi_{2}-\xi_{1} \sum_{i=1}^{m} L_{i}\left(u\left(t_{i}^{-}\right)\right)-\xi_{1} \sum_{i=1}^{m}\left(\rho \mathcal{J}_{\left(t_{i-1}\right)}^{1-\gamma+\alpha} \psi\right)\left(t_{i}\right) \\
& -\xi_{1}\left(\rho \mathcal{J}_{t_{m}^{+}}^{1-\gamma+\alpha} \psi\right)(b) .
\end{aligned}
$$

Now, taking $t=b$ in (43), we get

$$
\begin{aligned}
\left(\rho \mathcal{J}_{t_{m}^{+}}^{1-\gamma} u\right)(b)= & \xi_{2}+\left(1-\xi_{1}\right)\left(\sum_{i=1}^{m} L_{i}\left(u\left(t_{i}^{-}\right)\right)\right. \\
& \left.+\sum_{i=1}^{m}\left(\rho \mathcal{F}_{\left(t_{i-1}\right)^{+}}^{1-\gamma+\alpha}\right)\left(t_{i}\right)+\left(\rho \mathcal{F}_{t_{m}^{+}}^{1-\gamma+\alpha} \psi\right)(b)\right) .
\end{aligned}
$$

From (44) and (45), we find that

$$
c_{1}\left(\rho \mathscr{J}_{a^{+}}^{1-\gamma} u\right)\left(a^{+}\right)+c_{2}\left(\rho \mathcal{J}_{t_{m}^{+}}^{1-\gamma} u\right)(b)=c_{3}
$$

which shows that the boundary condition $c_{1}\left(\rho g_{a^{+}}^{1-\gamma} u\right)\left(a^{+}\right)$ $+c_{2}\left(\rho \mathcal{J}_{t_{m}^{+}}^{1-\gamma} u\right)(b)=c_{3}$ is satisfied. Next, apply operator ${ }^{\rho} D_{t_{k}^{+}}^{\gamma}$ on both sides of (34), where $k=0, \cdots, m$. Then, from Lemma 6 and Lemma 12, we obtain

$$
\left({ }^{\rho} D_{t_{k}^{+}}^{\gamma} u\right)(t)=\left({ }^{\rho} D_{t_{k}^{+}}^{\beta(1-\alpha)} \psi\right)(t)
$$

Since $u \in P C_{\gamma, \rho}^{\gamma}(J)$ and by definition of $P C_{\gamma, \rho}^{\gamma}(J)$, we have ${ }^{\rho} D_{t_{k}^{+}}^{\gamma} u \in P C_{\gamma, \rho}(J)$, then (47) implies that

$$
\left({ }^{\rho} D_{t_{k}^{+}}^{\gamma} u\right)(t)=\left(\delta_{\rho}^{\rho} \mathcal{F}_{t_{k}^{+}}^{1-\beta(1-\alpha)} \psi\right)(t)=\left({ }^{\rho} D_{t_{k}^{+}}^{\beta(1-\alpha)} \psi\right)(t) \in P C_{\gamma, \rho}(J)
$$

As $\psi(\cdot) \in C_{\gamma, \rho}(J)$ and from Lemma 4 , it follows that

$$
\left(\rho \mathcal{J}_{t_{k}^{+}}^{1-\beta(1-\alpha)} \psi\right) \in P C_{\gamma, \rho}(J)
$$

From (48) and (49) and by the definition of the space $P C_{\gamma, \rho}^{n}(J)$., we obtain

$$
\left(\rho \mathcal{J}_{t_{k}^{+}}^{1-\beta(1-\alpha)} \psi\right) \in P C_{\gamma, \rho}^{1}(J)
$$

Applying operator $\rho \mathscr{g}_{t_{k}^{+}}^{\beta(1-\alpha)}$ on both sides of (47) and using Lemma 8, Lemma 5, and Property 10, we have

$$
\begin{aligned}
\left({ }^{\rho} D_{t_{k}^{+}}^{\alpha, \beta} u\right)(t) & ={ }^{\rho} \mathscr{F}_{t_{k}^{+}}^{\beta(1-\alpha)}\left({ }^{\rho} D_{t_{k}^{+}}^{\gamma} u\right)(t) \\
& =\psi(t)-\frac{\left({ }^{\rho} \mathcal{F}_{t_{k}^{+}}^{1-\beta(1-\alpha)} \psi\right)\left(t_{k}\right)}{\Gamma(\beta(1-\alpha))}\left(\frac{t^{\rho}-t_{k}^{\rho}}{\rho}\right)^{\beta(1-\alpha)-1} \\
& =\psi(t),
\end{aligned}
$$


that is, (29) holds. Also, we can easily show that

$$
\left(\rho \mathcal{F}_{t_{k}^{+}}^{1-\gamma} u\right)\left(t_{k}^{+}\right)=\left(\rho \mathcal{F}_{t_{k-1}^{+}}^{1-\gamma} u\right)\left(t_{k}^{-}\right)+L_{k}\left(u\left(t_{k}^{-}\right)\right), k=1, \cdots, m
$$

This completes the proof.

As a consequence of Theorem 19, we have the following result.

Lemma 21. Let $\gamma=\alpha+\beta-\alpha \beta$, where $0<\alpha<1$ and $0 \leq \beta \leq 1$; let $f:(a, b] \times \mathbb{R} \times \mathbb{R} \rightarrow \mathbb{R}$ be a function such that $f(\cdot, u(\cdot), w$ $(\cdot)) \in C_{\gamma, \rho}(J)$ for any $u, w \in P C_{\gamma, \rho}(J)$. If $u \in P C_{\gamma, \rho}^{\gamma}(J)$, then $u$ satisfies problems ((2))-((4)) if and only if $u$ is the fixed point of the operator $\Psi: P C_{\gamma, \rho}(J) \rightarrow P C_{\gamma, \rho}(J)$ defined by

$$
\begin{aligned}
\Psi u(t)= & \frac{1}{\Gamma(\gamma)}\left(\frac{t^{\rho}-t_{k}^{\rho}}{\rho}\right)^{\gamma-1}\left[\xi_{2}-\xi_{1} \sum_{i=1}^{m} L_{i}\left(u\left(t_{i}^{-}\right)\right)\right. \\
& -\xi_{1} \sum_{i=1}^{m}\left(\rho \mathcal{F}_{\left(t_{i-1}\right)^{+}}^{1-\gamma+\alpha}\right)\left(t_{i}\right)-\xi_{1}\left(\rho g_{t_{m}^{+}}^{1-\gamma+\alpha} h\right)(b) \\
& \left.+\sum_{a<t_{k}<t} L_{k}\left(u\left(t_{i}^{-}\right)\right)+\sum_{a<t_{k}<t}\left(\rho \mathcal{F}_{\left(t_{k-1}\right)^{+}}^{1-\gamma+\alpha}\right)\left(t_{k}\right)\right] \\
& +\left({ }^{\rho} \mathcal{F}_{t_{k}^{+}}^{\alpha} h\right)(t), \quad t \in J_{k}, k=0, \cdots, m,
\end{aligned}
$$

where $h:(a, b] \rightarrow \mathbb{R}$ is a function satisfying the functional equation

$$
h(t)=f(t, u(t), h(t)) .
$$

Assume that the function $f:(a, b] \times \mathbb{R} \times \mathbb{R} \rightarrow \mathbb{R}$ is continuous and satisfies the following conditions:

(H1). The function $f:(a, b] \times \mathbb{R} \times \mathbb{R} \rightarrow \mathbb{R}$ be such that

$$
f(\cdot, u(\cdot), w(\cdot)) \in P C_{\gamma, \rho}^{\beta(1-\alpha)} \text {, for any } u, w \in P C_{\gamma, \rho}(J) .
$$

(H2). There exist constants $K>0$ and $0<M<1$ such that

$$
|f(t, u, w)-f(t, \bar{u}, \bar{w})| \leq K|u-\bar{u}|+M|w-\bar{w}|
$$

for any $u, w, \bar{u}, \bar{w} \in \mathbb{R}$ and $t \in(a, b]$.

(H3). There exists a constant $l^{*}>0$ such that

$$
\left|L_{k}(u)-L_{k}(\bar{u})\right| \leq l^{*}|u-\bar{u}|
$$

for any $u, \bar{u} \in \mathbb{R}$ and $k=1, \cdots, m$.

(H4). There exist functions $p_{1}, p_{2}, p_{3} \in C\left(J, \mathbb{R}_{+}\right)$with

$$
\begin{aligned}
& p_{1}^{*}=\sup _{t \in J} p_{1}(t), \\
& p_{2}^{*}=\sup _{t \in J} p_{2}(t), \\
& p_{3}^{*}=\sup _{t \in J} p_{3}(t)<1,
\end{aligned}
$$

such that

$|f(t, u, w)| \leq p_{1}(t)+p_{2}(t)|u|+p_{3}(t)|w|$, for $t \in(a, b], u, w \in \mathbb{R}$.

(H5). The functions $L_{k}: \mathbb{R} \rightarrow \mathbb{R}$ are continuous, and there exist constants $\Phi_{1}, \Phi_{2}>0$ such that

$$
\left|L_{k}(u)\right| \leq \Phi_{1}|u| \Phi_{2}, \quad \text { for each } u \in \mathbb{R}, k=1, \cdots, m \text {. }
$$

We are now in a position to state and prove our existence result for problems $((2))-((4))$ based on Banach's fixed point.

Theorem 22. Assume (H1)-(H3) hold. If

$$
\begin{aligned}
L:= & \left(\left|\xi_{1}\right|+1\right)\left(\frac{m l^{*} p^{*}}{\Gamma(\gamma)}+\frac{m K}{(1-M) \Gamma(1-\alpha)}\left(\frac{b^{\rho}-a^{\rho}}{\rho}\right)^{\alpha}\right) \\
& +\frac{K}{(1-M)}\left(\frac{\left|\xi_{1}\right|}{\Gamma(1-\alpha)}+\frac{\Gamma(\gamma)}{\Gamma(\gamma+\alpha)}\right)\left(\frac{b^{\rho}-a^{\rho}}{\rho}\right)^{\alpha}<1,
\end{aligned}
$$

then problems ((2))-((4)) have a unique solution in $P C_{\gamma, \rho}^{\gamma}(J)$ $\subset P C_{\gamma, \rho}^{\alpha, \beta}(J)$.

Proof. The proof will be given in two steps.

Step 1. We show that the operator $\Psi$ defined in (53) has a unique fixed point $u^{*}$ in $P C_{\gamma, \rho}(J)$. Let $u, w \in P C_{\gamma, \rho}(J)$ and $t$ $\epsilon(a, b]$; then, we have

$$
\begin{aligned}
& |\Psi u(t)-\Psi w(t)| \\
& \leq \frac{\left(t^{\rho}-t_{k}^{\rho}\right)^{\gamma-1}}{\Gamma(\gamma) \rho^{\gamma-1}}\left[\left|\xi_{1}\right| \sum_{i=1}^{m}\left|L_{i}\left(u\left(t_{i}^{-}\right)\right)-L_{i}\left(w\left(t_{i}^{-}\right)\right)\right|+\left|\xi_{1}\right|\right. \\
& \quad \times\left(\rho g_{t_{m}^{+}}^{1-\gamma+\alpha}|h(s)-g(s)|\right)(b)+\left|\xi_{1}\right| \sum_{i=1}^{m} \\
& \quad \times\left({ }^{\rho} g_{\left(t_{i-1}\right)^{+}}^{1-\gamma+\alpha}|h(s)-g(s)|\right)\left(t_{i}\right)+\sum_{a<t_{k}<t} \\
& \quad \times\left|L_{k}\left(u\left(t_{i}^{-}\right)\right)-L_{k}\left(w\left(t_{k}^{-}\right)\right)\right|+\sum_{a<t_{k}<t} \\
& \left.\quad \times\left(\rho g_{\left(t_{i-1}\right)^{+}}^{1-\gamma+\alpha}|h(s)-g(s)|\right)\left(t_{k}\right)\right]+\left(\rho \mathscr{J}_{t_{k}^{+}}^{\alpha}|h(s)-g(s)|\right)(t),
\end{aligned}
$$

where $h, g \in C_{\gamma, \rho}(J)$ such that

$$
\begin{aligned}
& h(t)=f(t, u(t), h(t)), \\
& g(t)=f(t, w(t), g(t)) .
\end{aligned}
$$

By $(\mathrm{H} 2)$, we have

$$
\begin{aligned}
|h(t)-g(t)| & =|f(t, u(t), h(t))-f(t, w(t), g(t))| \\
& \leq K|u(t)-w(t)|+M|h(t)-g(t)|
\end{aligned}
$$


Then,

$$
|h(t)-g(t)| \leq \frac{K}{1-M}|u(t)-w(t)| .
$$

Therefore, for each $t \in(a, b]$,

$$
\begin{aligned}
& |\Psi u(t)-\Psi w(t)| \\
& \leq \frac{\left(t^{\rho}-t_{k}^{\rho}\right)^{\gamma-1}}{\Gamma(\gamma) \rho^{\gamma-1}}\left[\left|\xi_{1}\right| \sum_{i=1}^{m} l^{*}\left|u\left(t_{i}\right)-w\left(t_{i}\right)\right|+\frac{\left|\xi_{1}\right| K}{1-M}\right. \\
& \quad \times\left(\rho \mathcal{F}_{t_{m}^{+}}^{1-\gamma+\alpha}|u(s)-w(s)|\right)(b)+\frac{\left|\xi_{1}\right| K}{1-M} \sum_{i=1}^{m} \\
& \quad \times\left(\rho \mathcal{F}_{\left(t_{i-1}\right)^{+}}^{1-\gamma+\alpha}|u(s)-w(s)|\right)\left(t_{i}\right)+\sum_{i=1}^{m} l^{*}\left|u\left(t_{i}\right)-w\left(t_{i}\right)\right| \\
& \left.\quad+\frac{K}{1-M} \sum_{i=1}^{m}\left(\rho \mathcal{F}_{\left(t_{i-1}\right)^{+}}^{1-\gamma+\alpha}|u(s)-w(s)|\right)\left(t_{i}\right)\right] \\
& \quad+\frac{K}{1-M} \sum_{i=1}^{m}\left(\rho \mathcal{F}_{t_{k}^{+}}^{\alpha}|u(s)-w(s)|\right)(t) .
\end{aligned}
$$

Thus,

$$
\begin{aligned}
\mid \Psi u(t) & -\Psi w(t) \mid \\
\leq & \frac{\left(t^{\rho}-t_{k}^{\rho}\right)}{\Gamma(\gamma) \rho^{\gamma-1}}\left[\left|\xi_{1}\right| m l^{*} p^{*}+\frac{\left|\xi_{1}\right| K}{1-M}\left(\rho \mathcal{F}_{t_{m}^{+}}^{1-\gamma+\alpha}\left(\frac{t^{\rho}-t_{m}^{\rho}}{\rho}\right)^{\gamma-1}\right)(b)\right. \\
& +\frac{m K\left|\xi_{1}\right|}{1-M}\left(\rho \mathcal{F}_{t_{k-1}}^{1-\gamma+\alpha}\left(\frac{s^{\rho}-t_{k-1}^{\rho}}{\rho}\right)^{\gamma-1}\right)\left(t_{k}\right)+m l^{*} p^{*}+\frac{m K}{1-M} \\
& \left.\times\left(\rho \mathcal{F}_{t_{k-1}}^{1-\gamma+\alpha}\left(\frac{s^{\rho}-t_{k-1}^{\rho}}{\rho}\right)^{\gamma-1}\right)\left(t_{k}\right)\right]+\frac{K}{1-M}\|u-w\|_{P C_{\gamma, \rho}} \\
& \times\left(\rho \mathcal{F}_{t_{k}}^{\alpha}\left(\frac{s^{\rho}-t_{k}^{\rho}}{\rho}\right)^{\gamma-1}\right)(t) .
\end{aligned}
$$

By Lemma 6, we have

$$
\begin{aligned}
\mid \Psi u(t) & -\Psi w(t) \mid \\
\leq & \frac{1}{\gamma}\left(\frac{t^{\rho}-t_{k}^{\rho}}{\rho}\right)^{\gamma-1}\|u-w\|_{P C_{\gamma, \rho}}\left[\left|\xi_{1}\right| m l^{*} p^{*}+\frac{\left|\xi_{1}\right| K \Gamma(\gamma)}{(1-M) \Gamma(1-\alpha)}\right. \\
& \times\left(\frac{b^{\rho}-b_{m}^{\rho}}{\rho}\right)^{\alpha}+\frac{m K\left|\xi_{1}\right| \Gamma(\gamma)}{(1-M) \Gamma(1-\alpha)}\left(\frac{t_{k}^{\rho}-t_{k-1}^{\rho}}{\rho}\right)^{\alpha}+m l^{*} p^{*} \\
& \left.+\frac{m K \Gamma(\gamma)}{(1-M) \Gamma(1-\alpha)}\left(\frac{t_{k}^{\rho}-t_{k-1}^{\rho}}{\rho}\right)^{\alpha}\right]+\frac{K \Gamma(\gamma)}{(1-M) \Gamma(\gamma-\alpha)} \\
& \times\|u-w\|_{P C_{\gamma, \rho}}\left(\frac{t^{\rho}-t_{k}^{\rho}}{\rho}\right)^{\alpha+\gamma-1} .
\end{aligned}
$$

Hence,

$$
\begin{aligned}
& \left|\left(\frac{t^{\rho}-t_{k}^{\rho}}{\rho}\right)^{1-\gamma}(\Psi u(t)-\Psi w(t))\right| \\
& \leq\left[\left(\left|\xi_{1}\right|+1\right)\left(\frac{m l^{*} p^{*}}{\Gamma(\gamma)}+\frac{m K}{(1-M) \Gamma(1+\alpha)}\left(\frac{b^{\rho}-a^{\rho}}{\rho}\right)^{\alpha}\right)\right. \\
& \left.\quad+\frac{K}{(1-M)}\left(\frac{\left|\xi_{1}\right|}{\Gamma(1+\alpha)}+\frac{\Gamma(\gamma)}{\Gamma(\gamma+\alpha)}\right)\left(\frac{b^{\rho}-a^{\rho}}{\rho}\right)^{\alpha}\right] \\
& \quad \times\|u-w\|_{P C_{\gamma, \rho}}
\end{aligned}
$$

which implies that

$$
\begin{aligned}
\| \Psi u- & \Psi w \|_{P C_{\gamma, \rho}} \\
\leq & {\left[\left(\left|\xi_{1}\right|+1\right)\left(\frac{m l^{*} p^{*}}{\Gamma(\gamma)}+\frac{m K}{(1-M) \Gamma(1+\alpha)}\left(\frac{b^{\rho}-a^{\rho}}{\rho}\right)^{\alpha}\right)\right.} \\
& \left.+\frac{K}{(1-M)}\left(\frac{\left|\xi_{1}\right|}{\Gamma(1+\alpha)}+\frac{\Gamma(\gamma)}{\Gamma(\gamma+\alpha)}\left(\frac{b^{\rho}-a^{\rho}}{\rho}\right)^{\alpha}\right)\right] \\
& \times\|u-w\|_{P C_{\gamma, \rho}} .
\end{aligned}
$$

By (61), the operator $\Psi$ is a contraction. Hence, by Theorem 14, $\Psi$ has a unique fixed point $u^{*} \in P C_{\gamma, \rho}(J)$.

Step 2. We show that such a fixed point $u^{*} \in P C_{\gamma, \rho}(J)$ is actually in $P C_{\gamma, \rho}^{\gamma}(J)$.

Since $u^{*}$ is the unique fixed point of operator $\Psi$ in $P C_{\gamma, \rho}(J)$, then for each $t \in J_{k}$, with $k=0, \cdots, m$, we have

$$
\begin{aligned}
u^{*}(t)= & \frac{1}{\Gamma(\gamma)}\left(\frac{t^{\rho}-t_{k}^{\rho}}{\rho}\right)^{\gamma-1}\left[\xi_{2}-\xi_{1} \sum_{i=1}^{m} L_{i}\left(u\left(t_{i}^{-}\right)\right)-\xi_{1} \sum_{i=1}^{m}\right. \\
& \cdot\left(\rho \mathcal{F}_{\left(t_{i-1}\right)^{+}}^{1-\gamma+\alpha}\right)\left(t_{i}\right)-\xi_{1}\left(\rho \mathcal{F}_{t_{m}^{+}-\gamma+\alpha}^{1-\alpha} h\right)(b)+\sum_{a<t_{k}<t}\left(u\left(t_{i}^{-}\right)\right) \\
& \left.+\sum_{a<t_{k}<t}\left(\rho \mathcal{F}_{\left(t_{i-1}\right)^{+}}^{1-\gamma+\alpha}\right)\left(t_{k}\right)\right]+\left(\rho \mathcal{F}_{t_{k}^{+}}^{\alpha} h\right)(t),
\end{aligned}
$$

where $h \in C_{\gamma, \rho}(J)$ such that

$$
h(t)=f\left(t, u^{*}(t), h(t)\right) .
$$

Applying ${ }^{\rho} D_{t_{k}^{+}}^{\gamma}$ to both sides and by Lemma 6 and Lemma 12 , we get

$$
\begin{aligned}
{ }^{\rho} D_{t_{k}^{+}}^{\gamma} u^{*}(t) & =\left({ }^{\rho} D_{t_{k}^{+}}^{\gamma} \mathcal{F}_{t_{k}^{+}}^{\alpha} f\left(s, u^{*}(s), h(s)\right)\right)(t) \\
& =\left({ }^{\rho} D_{t_{k}^{+}}^{\beta(1-\alpha)} f\left(s, u^{*}(s), h(s)\right)\right)(t) .
\end{aligned}
$$

Since $\gamma \geq \alpha$, by (H1), the right-hand side is in $P C_{\gamma, \rho}(J)$ and thus, ${ }^{\rho} D_{t_{k}^{+}}^{\gamma} u^{*} \in P C_{\gamma, \rho}(J)$ which implies that $u^{*} \in P C_{\gamma, \rho}^{\gamma}(J)$. 
As a consequence of Steps 1 and 2 together with Theorem 22, we can conclude that problems $((2))-((4))$ have a unique solution in $P C_{\gamma, \rho}^{\gamma}(J)$.

Our second result is based on Schaefer's fixed-point theorem.

Theorem 23. Assume (H1), (H4), and (H5) hold. If

$$
\begin{aligned}
\left(\left|\xi_{1}\right|\right. & +1)\left(\frac{m \Phi_{1} p^{*}}{\Gamma(\gamma)}+\frac{m p_{2}^{*}\left(b^{\rho}-a^{\rho}\right)^{\alpha}}{\left(1-p_{3}^{*}\right) \Gamma(1+\alpha) \rho^{\alpha}}\right) \\
& +\left(\frac{\left|\xi_{1}\right|}{\Gamma(1+\alpha)}+\frac{\Gamma(\gamma)}{\Gamma(\gamma+\alpha)}\right)\left(\frac{p_{2}^{*}\left(b^{\rho}-a^{\rho}\right)^{\alpha}}{\left(1-p_{3}^{*}\right) \rho^{\alpha}}\right)<1
\end{aligned}
$$

then problems ((2))-((4)) have at least one solution in $P C_{\gamma, \rho}^{\gamma}(J) \subset P C_{\gamma, \rho}^{\alpha, \beta}(J)$.

Proof. We shall use Schaefer's fixed-point theorem to prove in several steps that the operator $\Psi$ defined in (53) has a fixed point.

Step 1. $\Psi$ is continuous. Let $\left\{u_{n}\right\}$ be a sequence such that $u_{n} \rightarrow u$ in $P C_{\gamma, \rho}(J)$. Then, for each $t \in(a, b]$, we have

$$
\begin{aligned}
&\left|\left(\left(\Psi u_{n}\right)(t)-(\Psi u)(t)\right)\left(\frac{t^{\rho}-t_{k}^{\rho}}{\rho}\right)^{1-\gamma}\right| \\
& \leq \frac{1}{\Gamma(\gamma)}\left[\left|\xi_{1}\right| \sum_{i=1}^{m}\left|L_{i}\left(u_{n}\left(t_{i}^{-}\right)\right)-L_{i}\left(u\left(t_{i}^{-}\right)\right)\right|+\left|\xi_{1}\right|\right. \\
& \times\left({ }^{\rho} g_{t_{m}^{+}}^{1-\gamma+\alpha}\left|h_{n}(s)-h(s)\right|\right)(b)+\left|\xi_{1}\right| \sum_{i=1}^{m} \\
& \times\left({ }^{\rho} g_{\left(t_{i-1}\right)^{+}}^{1-\gamma+\alpha}\left|h_{n}(s)-h(s)\right|\right)\left(t_{i}\right)+\sum_{a<t_{k}<t} \\
& \times\left|L_{k}\left(u_{n}\left(t_{k}^{-}\right)\right)-L_{k}\left(u\left(t_{k}^{-}\right)\right)\right|+\sum_{a<t_{k}<t} \\
&\left.\times\left({ }^{\rho} \mathcal{g}_{\left(t_{i-1}\right)^{1-\gamma+\alpha}}^{1-\alpha} h_{n}(s)-h(s) \mid\right)\left(t_{k}\right)\right]+\left(\frac{t^{\rho}-t_{k}^{\rho}}{\rho}\right)^{1-\gamma} \\
& \times\left({ }^{\rho} g_{t_{k}^{+}}^{\alpha}\left|h_{n}(s)-h(s)\right|\right)(t),
\end{aligned}
$$

where $h_{n}, h \in C_{\gamma, \rho}(J)$ such that

$$
\begin{aligned}
h_{n}(t) & =f\left(t, u_{n}(t), h_{n}(t)\right), \\
h(t) & =f(t, u(t), h(t)) .
\end{aligned}
$$

Since $u_{n} \rightarrow u$, then we get $h_{n}(t) \rightarrow h(t)$ as $n \rightarrow \infty$, for each $t \in(a, b]$, and since $f$ and $L_{k}$ are continuous, then we have

$$
\left\|\Psi u_{n}-\Psi u\right\|_{P C_{\gamma, \rho}} \rightarrow 0 \text { as } n \rightarrow \infty
$$

Step 2. We show that $\Psi$ maps bounded set into bounded set of $P C_{\gamma, \rho}(J)$. For $\eta>0$, there exists a positive constant $r$ such that for $u \in B_{\eta}=\left\{u \in P C_{\gamma, \rho}(J):\|u\|_{P C_{\gamma, \rho}} \leq \eta\right\}$, we have $\|\Psi(u)\|_{P C_{\gamma, \rho}} \leq r$.

By (H4) and from (53), we have for each $t \in J_{k}, k=0$, $\cdots, m$,

$$
\begin{aligned}
& \left|\left(\frac{t^{\rho}-t_{k}^{\rho}}{\rho}\right)^{1-\gamma} h(t)\right| \\
& \quad=\left|\left(\frac{t^{\rho}-t_{k}^{\rho}}{\rho}\right)^{1-\gamma} f(t, u(t), h(t))\right| \\
& \quad \leq\left(\frac{t^{\rho}-t_{k}^{\rho}}{\rho}\right)^{1-\gamma}\left(p_{1}(t)+p_{2}(t)|u(t)|+p_{3}(t)|h(t)|\right),
\end{aligned}
$$

which implies that

$$
\|h\|_{P C_{\gamma, \rho}} \leq p_{1}^{*}\left(\frac{b^{\rho}-a^{\rho}}{\rho}\right)^{1-\gamma}+p_{2}^{*} \eta+p_{3}^{*}\|h\|_{P C_{\gamma, \rho}} .
$$

Then,

$$
\|h\|_{P C_{\gamma, \rho}} \leq \frac{p_{1}^{*}\left(\left(b^{\rho}-a^{\rho}\right) / \rho\right)^{1-\gamma}+p_{2}^{*} \eta}{1-p_{3}^{*}}:=\Lambda .
$$

Thus, (53) implies

$$
\begin{aligned}
& \left|\left(\frac{t^{\rho}-t_{k}^{\rho}}{\rho}\right)^{1-\gamma}(\Psi u)(t)\right| \\
& \leq \frac{1}{\Gamma(\gamma)}\left[\left|\xi_{2}\right|+\left|\xi_{1}\right| \sum_{i=1}^{m}\left|L_{i}(u)\left(t_{i}^{-}\right)\right|+\left|\xi_{1}\right| \sum_{i=1}^{m}\left(\rho \mathcal{g}_{\left(t_{i-1}\right)^{+}}^{1-\gamma+\alpha}|h(s)|\right)\left(t_{i}\right)\right. \\
& +\left|\xi_{1}\right|\left({ }^{\rho} \mathcal{g}_{t_{m}^{1+}}^{1-\gamma+\alpha}|h(s)|\right)(b)+\sum_{a<t_{k}<t}\left|L_{k}\left(u\left(t_{i}^{-}\right)\right)\right|+\sum_{a<t_{k}<t} \\
& \left.\times\left(\rho \mathcal{g}_{\left(t_{i-1}\right)^{+}}^{1-\gamma+\alpha}|h(s)|\right)\left(t_{k}\right)\right]+\left(\frac{t^{\rho}-t_{k}^{\rho}}{\rho}\right)^{1-\gamma}\left(\rho \mathcal{J}_{t_{k}^{+}}^{\alpha}|h(s)|\right)(t), \\
& \leq \frac{1}{\Gamma(\gamma)}\left[\left|\xi_{2}\right|+\left|\xi_{1}\right| m\left(\Phi_{1} p^{*} \eta+\Phi_{2}\right)+\left|\xi_{1}\right| m \Lambda\right. \\
& \times\left(\rho \mathcal{g}_{\left(t_{i-1}\right)^{+}}^{1-\gamma+\alpha}\left(\frac{s^{\rho}-t_{k-1}^{\rho}}{\rho}\right)^{\gamma-1}\right)\left(t_{k}\right)+\left|\xi_{1}\right| \Lambda \\
& \times\left(\rho \mathcal{F}_{t_{m}^{+}}^{1-\gamma+\alpha}\left(\frac{s^{\rho}-t_{m}^{\rho}}{\rho}\right)^{\gamma-1}\right)(b)+m\left(\Phi_{1} p^{*} \eta+\Phi_{2}\right)+m \Lambda \\
& \left.\times\left(\rho \mathcal{J}_{\left(t_{i-1}\right)^{+}}^{1-\gamma+\alpha}\left(\frac{s^{\rho}-t_{k-1}^{\rho}}{\rho}\right)^{\gamma-1}\right)\left(t_{k}\right)\right]+\Lambda\left(\frac{t^{\rho}-t_{k}^{\rho}}{\rho}\right)^{1-\gamma} \\
& \times\left(\rho \mathcal{F}_{t_{k}^{+}}^{\alpha}\left(\frac{s^{\rho}-t_{k}^{\rho}}{\rho}\right)^{\gamma-1}\right)(t) \text {. }
\end{aligned}
$$


By Lemma 6, we have

$$
\begin{aligned}
\|\Psi u\|_{P C_{\gamma, \rho} \leq} \leq & \left(\left|\xi_{1}\right|+1\right)\left(\frac{m\left(\Phi_{1} p^{*} \eta+\Phi_{2}\right)}{\Gamma(\gamma)}+\frac{m \Lambda}{\Gamma(1+\alpha)}\left(\frac{b^{\rho}-a^{\rho}}{\rho}\right)^{\alpha}\right. \\
& \left.+\Lambda\left(\frac{\left|\xi_{1}\right|}{\Gamma(1+\alpha)}+\frac{\Gamma(\gamma)}{\Gamma(\gamma+\alpha)}\right)\left(\frac{b^{\rho}-a^{\rho}}{\rho}\right)^{\alpha}+\frac{\left|\xi_{2}\right|}{\Gamma(\gamma)}\right) .
\end{aligned}
$$

Step 3. $\Psi$ maps bounded sets into equicontinuous sets of $P C_{\gamma, \rho}$.

Let $\epsilon_{1}, \epsilon_{2} \in J, \epsilon_{1}<\epsilon_{2} B_{\eta}$, be a bounded set of $P C_{\gamma, \rho}$ as in Step 2, and let $u \in B_{\eta}$.

Then,

$$
\begin{aligned}
& \left|\left(\frac{\epsilon_{1}^{\rho}-t_{k}^{\rho}}{\rho}\right)^{1-\gamma}(\Psi u)\left(\epsilon_{1}\right)-\left(\frac{\epsilon_{2}^{\rho}-t_{k}^{\rho}}{\rho}\right)^{1-\gamma}(\Psi u)\left(\epsilon_{2}\right)\right| \\
& \leq \frac{1}{\Gamma(\gamma)}\left[\sum_{\epsilon_{1}<t_{k}<\epsilon_{2}}\left|L_{k}\left(u\left(t_{i}^{-}\right)\right)\right|+\sum_{\epsilon_{1}<t_{k}<\epsilon_{2}}\left(\rho \mathcal{F}_{\left(t_{i-1}\right)}^{1-\gamma+\alpha}|h(s)|\right)\left(t_{k}\right)\right] \\
& \quad+\frac{\Lambda \Gamma(\gamma)}{\Gamma(\gamma+\alpha)}\left|\left(\frac{\epsilon_{1}^{\rho}-t_{k}^{\rho}}{\rho}\right)^{\alpha}-\left(\frac{\epsilon_{2}^{\rho}-t_{k}^{\rho}}{\rho}\right)^{\alpha}\right| .
\end{aligned}
$$

As $\epsilon_{1} \rightarrow \epsilon_{2}$, the right-hand side of the above inequality tends to zero. From Steps 1 to 3 with Arzela-Ascoli theorem, we conclude that $\Psi: P C_{\gamma, \rho} \rightarrow P C_{\gamma, \rho}$ is continuous and completely continuous.

Step 4. A priori bound. Now it remains to show that the set

$$
G=\left\{u \in P C_{\gamma, \rho}: u=\lambda * \Psi(u), \quad \text { for some } 0<\lambda^{*}<1\right\} \text {, }
$$

is bounded. Let $u \in G$, then $u=\lambda^{*} \Psi(u)$ for some $0<$ $\lambda^{*}<1$
By (H4), we have for each $t \in J$

$$
\begin{aligned}
\left|\left(\frac{t^{\rho}-t_{k}^{\rho}}{\rho}\right)^{1-\gamma} h(t)\right| & =\left|\left(\frac{t^{\rho}-t_{k}^{\rho}}{\rho}\right)^{1-\gamma} f(t, u(t), h(t))\right| \\
& \leq\left(\frac{t^{\rho}-t_{k}^{\rho}}{\rho}\right)^{1-\gamma}\left(p_{1}(t)+p_{2}(t)|u(t)|+p_{3}(t)|h(t)|\right),
\end{aligned}
$$

which implies that

$$
\|h\|_{P C_{\gamma, \rho}} \leq p_{1}^{*}\left(\frac{b^{\rho}-a^{\rho}}{\rho}\right)^{1-\gamma}+p_{2}^{*}\|u\|_{P C_{\gamma, \rho}}+p_{3}^{*}\|h\|_{P C_{\gamma, \rho}},
$$

then

$$
\|h\|_{P C_{\gamma, \rho}} \leq \frac{p_{1}^{*}\left(\left(b^{\rho}-a^{\rho}\right) / \rho\right)^{1-\gamma}+p_{2}^{*}\|u\|_{P C_{\gamma, \rho}}}{1-p_{3}^{*}} .
$$

This implies, by (53) and (H5) and by letting the estimation of Step 2, that for each $t \in J$, we have

$$
\begin{aligned}
\|u\|_{P C_{\gamma, \rho}} \leq & \left(\left|\xi_{1}\right|+1\right)\left(\frac{m\left(\Phi_{1} p^{*}\|u\|_{P C_{\gamma, \rho}}+\Phi_{2}\right)}{\Gamma(\gamma)}\right. \\
& \left.+\frac{m p_{1}^{*}\left(b^{\rho}-a^{\rho} / \rho\right)^{1+\alpha-\gamma}+m p_{2}^{*}\|u\|_{P C_{\gamma, \rho}}\left(b^{\rho}-a^{\rho} / \rho\right)^{\alpha}}{\left(1-p_{3}^{*}\right) \Gamma(1-\alpha)}\right) \\
& +\frac{p_{1}^{*}\left(b^{\rho}-a^{\rho} / \rho\right)^{1+\alpha-\gamma}+p_{2}^{*}\|u\|_{P C_{\gamma, \rho}}\left(b^{\rho}-a^{\rho} / \rho\right)^{\alpha}}{\left(1-p_{3}^{*}\right)} \\
& \cdot\left(\frac{\left|\xi_{1}\right|}{\Gamma(1-\alpha)}+\frac{\Gamma(\gamma)}{\Gamma(1-\alpha)}\right)+\frac{\left|\xi_{2}\right|}{\Gamma(\gamma)}, \\
\leq & {\left[\left(\left|\xi_{1}\right|+1\right)\left(\frac{m \Phi_{1} p^{*}}{\Gamma(\gamma)}+\frac{m p_{2}^{*}\left(b^{\rho}-a^{\rho}\right)^{\alpha}}{\left(1-p_{3}^{*}\right) \Gamma(1+\alpha) \rho^{\alpha}}\right)\right.} \\
& \left.+\frac{\left|\xi_{1}\right|}{\Gamma(1+\alpha)}+\frac{\Gamma(\gamma)}{\Gamma(\gamma+\alpha)}\left(\frac{p_{2}^{*}\left(b^{\rho}-a^{\rho}\right)^{\alpha}}{\left(1-p_{3}^{*}\right) \rho^{\alpha}}\right)\right]\|u\|_{P C_{\gamma, \rho}} \\
& +\frac{\left|\xi_{2}\right|}{\Gamma(\gamma)}\left(\left|\xi_{1}\right|+1\right)\left(\frac{m \Phi_{2}}{\Gamma(\gamma)}+\frac{m p_{1}^{*}\left(b^{\rho}-a^{\rho}\right)^{1+\alpha-\gamma}}{\left(1-p_{3}^{*}\right) \Gamma(1+\alpha) \rho^{1+\alpha-\gamma}}\right) \\
& +\frac{\left|\xi_{1}\right|}{\Gamma(1+\alpha)} \frac{\Gamma(\gamma)}{\Gamma(\gamma+\alpha)}\left(\frac{p_{1}^{*}\left(b^{\rho}-a^{\rho}\right)^{1+\alpha-\gamma}}{\left(1-p_{3}^{*}\right) \rho^{1+\alpha-\gamma}}\right) .
\end{aligned}
$$

By (74), we have

$$
\begin{aligned}
& \|u\|_{P C_{\gamma \rho}} \\
& \quad \leq \frac{\left[\left(\left|\xi_{2}\right|+1\right)\left(\left(m \Phi_{2} / \Gamma(\gamma)\right)\left(m p_{1}^{*}\left(b^{\rho}-a^{\rho}\right)^{1+\alpha-\gamma} /\left(1-p_{3}^{*}\right) \Gamma(1+\alpha) \rho^{1+\alpha-\gamma}\right)\right)+\left(\left(\left|\xi_{1}\right| / \Gamma(1+\alpha)\right)+(\Gamma(\gamma) / \Gamma(\lambda+\alpha))\right)\left(p_{1}^{*}\left(b^{\rho}-a^{\rho}\right)^{1+\alpha-\gamma} /\left(1-p_{3}^{*}\right) \rho^{1+\alpha-\gamma}\right)\right]}{1-\left[\left(\left|\xi_{1}\right|+1\right)\left(\left(m \Phi_{1} p^{*} / \Gamma(\gamma)\right)+\left(m p_{2}^{*}\left(b^{\rho}-a^{\rho}\right)^{\alpha} /\left(1-p_{3}^{*}\right) \Gamma(1+\alpha) \rho^{\alpha}\right)\right)+\left(\left(\left|\xi_{1}\right| / \Gamma(1+\alpha)\right)+(\Gamma(\gamma) / \Gamma(\lambda+\alpha))\right)\left(p_{2}^{*}\left(b^{\rho}-a^{\rho}\right)^{\alpha} /\left(1-p_{3}^{*}\right) \rho^{\alpha}\right)\right]}:=R .
\end{aligned}
$$


As a consequence of Theorem 15 and using Step 2, we deduce that $\Psi$ has a fixed point which is a solution of problems (2)-(4).

Our third result is based on Krasnoselskii's fixed-point theorem.

Theorem 24. Assume (H1), (H4), and (H5) hold. If

$$
\begin{gathered}
\left(\left|\xi_{1}\right| \mid+1\right)\left(\frac{m \Phi_{1} p^{*}}{\Gamma(\gamma)}+\frac{m p_{2}^{*}\left(b^{\rho}-a^{\rho}\right)^{\alpha}}{\left(1-p_{3}^{*}\right) \Gamma(1+\alpha) \rho^{\alpha}}\right) \\
+\frac{p_{2}^{*}\left|\xi_{1}\right|\left(b^{\rho}-a^{\rho}\right)^{\alpha}}{\left(1-p_{3}^{*}\right) \Gamma(1+\alpha) \rho^{\alpha}}<1
\end{gathered}
$$

then problems ((2))-((4)) have at least one solution in $P C_{\gamma, \rho}^{\gamma}(J) \subset P C_{\gamma, \rho}^{\alpha, \beta}(J)$.

Proof. Consider the set

$$
B_{\eta}=\left\{u \in P C_{\gamma, \rho}(J):\|u\|_{P C_{\gamma, \rho}} \leq \eta\right\}
$$

$\eta \geq \frac{\left(\left|\xi_{1}\right|+1\right)\left(\left(m \Phi_{2} / \Gamma(\gamma)\right)+\left(m \Phi_{2} / \Gamma(1+\alpha)\right)\left(\left(b^{\rho}-a^{\rho}\right) / \rho\right)^{\alpha}\right)+\Lambda\left(\left(\left|\xi_{1}\right| / \Gamma(1+\alpha)\right)+(\Gamma(\gamma) / \Gamma(\gamma+\alpha))\right)\left(\left(b^{\rho}-a^{\rho}\right) / \rho\right)^{\alpha}\left(\left|\xi_{2}\right| / \Gamma(\gamma)\right)}{1-\left(\left|\xi_{1}\right|+1\right)\left(m \Phi_{1} p^{*} / \Gamma(\gamma)\right)}$

We define the operators $Q_{1}$ and $Q_{2}$ on $B_{\eta}$ by

$$
\begin{aligned}
Q_{1} u(t)= & \frac{1}{\Gamma(\gamma)}\left(\frac{\left(t^{\rho}-t_{k}^{\rho}\right)}{\rho}\right)^{\gamma-1}\left[\xi_{2}-\xi_{1} \sum_{i=1}^{m} L_{i}\left(u\left(t_{i}^{-}\right)\right)\right. \\
& -\xi_{1} \sum_{i=1}^{m}\left(\rho \mathcal{F}_{\left(t_{i-1}\right)}^{1-\gamma+\alpha}\right)\left(t_{i}\right)-\xi_{1}\left(\rho \mathcal{F}_{t_{m}^{+}}^{1-\gamma+\alpha} h\right)(b) \\
& \left.+\sum_{a<t_{k}<t} L_{k}\left(u\left(t_{k}^{-}\right)\right)+\sum_{a<t_{k}<t}\left(\rho \mathcal{F}_{\left(t_{k-1}\right)}^{1-\gamma+\alpha} h\right)\left(t_{k}\right)\right],
\end{aligned}
$$

$$
Q_{2} u(t)=\left(\rho \mathcal{F}_{t_{k}^{+}}^{\alpha} h\right)(t)
$$

where $k=0, \cdots, m$, and $h:(a, b] \rightarrow \mathbb{R}$ be a function satisfying the functional equation

$$
h(t)=f(t, u(t), h(t)) .
$$

Then, the fractional integral equation (53) can be written as operator equation

$$
\Psi u(t)=Q_{1} u(t)+Q_{2} u(t), \quad u \in P C_{\gamma, \rho}(J)
$$

The proof will be given in several steps.

Step 1. We prove that $Q_{1} u+Q_{2} w \in B_{\eta}$, for any $u, z \in B_{\eta}$.

By (H4), (H5), and Lemma 6 , for each $t \in(a, b]$, we have

$$
\begin{aligned}
&\left\|Q_{1} u+Q_{2} w\right\|_{P C_{\gamma, \rho}} \\
& \leq\left\|Q_{1} u\right\|_{P C_{\gamma, \rho}}+\left\|Q_{2} u\right\|_{P C_{\gamma, \rho}} \\
& \leq\left(\left|\xi_{1}\right|+1\right)\left(\frac{m\left(\Phi_{1} p^{*} \eta+\Phi_{2}\right)}{\Gamma(\gamma)}+\frac{m \Lambda}{\Gamma(1+\alpha)}\left(\frac{b^{\rho}-a^{\rho}}{\rho}\right)^{\alpha}\right) \\
&+\Lambda\left(\frac{\left|\xi_{1}\right|}{\Gamma(1+\alpha)}+\frac{\Gamma(\gamma)}{\Gamma(\gamma+\alpha)}\right)\left(\frac{b^{\rho}-a^{\rho}}{\rho}\right)^{\alpha} \frac{\left|\xi_{2}\right|}{\Gamma(\gamma)} .
\end{aligned}
$$

Since

$$
\eta \geq \frac{\left(\left|\xi_{1}\right|+1\right)\left(m \Phi_{2} / \Gamma(\gamma)\right)+(m \Lambda / \Gamma(1+\alpha))\left(\left(b^{\rho}-a^{\rho}\right) / \rho\right)^{\alpha}+\Lambda\left(\left|\xi_{1}\right| / \Gamma(1+\alpha)+(\Gamma(\gamma) / \Gamma(\gamma+\alpha))\right)\left(\left(b^{\rho}-a^{\rho}\right) / \rho\right)^{\alpha}+\left(\left|\xi_{2}\right| / \Gamma(\gamma)\right)}{1-\left(\left|\xi_{1}\right|+1\right)\left(m \Phi_{1} p^{*} / \Gamma(\gamma)\right)},
$$


we have

$$
\left\|Q_{1} y+Q_{2} z\right\|_{P C_{\gamma, \rho}} \leq \eta
$$

which infers that $Q_{1} u+Q_{2} w \in B_{\eta}$.

Step 2. $Q_{1}$ is a contraction.

Let $u, w \in P C_{\gamma, \rho}(J)$ and $t \in(a, b]$.

By (H4), we have

$$
\begin{aligned}
|h(t)-g(t)| & =|f(t, u(t), h(t))-f(t, w(t), g(t))| \\
& \leq p_{2}(t)|u(t)-w(t)|+p_{3}(t)|h(t)-g(t)|
\end{aligned}
$$

Then,

$|h(t)-g(t)| \leq \frac{p_{2}(t)}{1-p_{3}(t)}|u(t)-w(t)| \leq \frac{p_{2}^{*}(t)}{1-p_{3}^{*}(t)}|u(t)-w(t)|$,

where $p_{1}^{*}=\sup _{t \in J} p_{1}(t), p_{2}^{*}=\sup _{t \in J} p_{2}(t)$, and $h, g \in C_{\gamma, \rho}(J)$ such that

$$
\begin{aligned}
& h(t)=f(t, u(t), h(t)), \\
& g(t)=f(t, w(t), g(t)) .
\end{aligned}
$$

Then, by (H5), we have

$$
\begin{aligned}
& \left|Q_{1} y(t)-Q_{1} z(t)\right| \\
& \leq \frac{1}{\Gamma(\gamma)}\left(\frac{t^{\rho}-t_{k}^{\rho}}{\rho}\right)^{\gamma-1}\|u-w\|_{P C_{\gamma, \rho}} \\
& \quad \times\left[\left|\xi_{1}\right| m \Phi_{1} p^{*}+\frac{p_{2}^{*}\left|\xi_{1}\right| \Gamma(\gamma)}{\left(1-p_{3}^{*}\right) \Gamma(1-\alpha)}\left(\frac{b^{\rho}-t_{m}^{\rho}}{\rho}\right)^{\alpha}\right. \\
& \quad+\frac{m p_{2}^{*}\left|\xi_{1}\right| \Gamma(\gamma)}{\left(1-p_{3}^{*}\right) \Gamma(1-\alpha)}\left(\frac{t_{k}^{\rho}-t_{k-1}^{\rho}}{\rho}\right)^{\alpha}+m \Phi_{1} p^{*} \\
& \left.\quad+\frac{m p_{2}^{*} \Gamma(\gamma)}{\left(1-p_{3}^{*}\right) \Gamma(1-\alpha)}\left(\frac{t_{k}^{\rho}-t_{k-1}^{\rho}}{\rho}\right)^{\alpha}\right]
\end{aligned}
$$

Hence,

$$
\begin{aligned}
& \left\|Q_{1} u-Q_{1} w\right\|_{P C_{\gamma, \rho}} \\
& \leq\left[\left(\left|\xi_{1}\right|+1\right)\left(\frac{m \Phi_{1} p^{*}}{\Gamma(\gamma)}+\frac{m p_{2}^{*}}{\left(1-p_{3}^{*}\right) \Gamma(1+\alpha)}\left(\frac{b^{\rho}-a^{\rho}}{\rho}\right)^{\alpha}\right)\right. \\
& \left.\quad+\frac{p_{2}^{*}\left|\xi_{1}\right|}{\left(1-p_{3}^{*}\right) \Gamma(1+\alpha)}\left(\frac{b^{\rho}-a^{\rho}}{\rho}\right)^{\alpha}\right]\|u-w\|_{P C_{\gamma, \rho}}
\end{aligned}
$$

By (90), the operator $Q_{1}$ is a contraction.
Step 3. $Q_{2}$ is continuous and compact.

The continuity of $Q_{2}$ follows from the continuity of $f$. Next, we prove that $Q_{2}$ is uniformly bounded on $B_{\eta}$. Let any $w \in B_{\eta}$. (94) imply

$$
\begin{aligned}
\left|\left(\frac{t^{\rho}-t_{k}^{\rho}}{\rho}\right)^{1-\gamma}\left(Q_{2} z\right)(t)\right| & \leq\left(\frac{t^{\rho}-t_{k}^{\rho}}{\rho}\right)^{1-\gamma}\left(\rho \mathcal{F}_{t_{k}^{+}}^{\alpha}|g(s)|\right)(t), \\
& \leq \Lambda\left(\frac{t^{\rho}-t_{k}^{\rho}}{\rho}\right)^{1-\gamma}\left(\rho \mathcal{F}_{t_{k}^{+}}^{\alpha}\left(\frac{s^{\rho}-t_{k}^{\rho}}{\rho}\right)^{\gamma-1}\right)(t),
\end{aligned}
$$

where $k=0, \cdots, m$, and $h:(a, b] \rightarrow \mathbb{R}$ is a function satisfying the functional equation

$$
g(t)=f(t, w(t), g(t))
$$

By Lemma 6, we have

$$
\left\|Q_{2} z\right\|_{P C_{\gamma, \rho}} \leq \frac{\Lambda \Gamma(\gamma)}{\Gamma(\gamma+\alpha)}\left(\frac{b^{\rho}-a^{\rho}}{\rho}\right)^{\alpha}
$$

This means that $Q_{2}$ is uniformly bounded on $B_{\eta}$. Next, we show that $Q_{2} B_{\eta}$ is equicontinuous. Let any $w \in B_{\eta}$ and $a<$ $\epsilon_{1}<\epsilon_{2} \leq b$. Then,

$$
\begin{aligned}
& \left|\left(\frac{\epsilon_{1}^{\rho}-t_{k}^{\rho}}{\rho}\right)^{1-\gamma}\left(Q_{2} z\right)\left(\epsilon_{1}\right)-\left(\frac{\epsilon_{2}^{\rho}-t_{k}^{\rho}}{\rho}\right)^{1-\gamma}\left(Q_{2} z\right)\left(\epsilon_{2}\right)\right| \\
& \quad \leq \frac{\Lambda \Gamma(\gamma)}{\Gamma(\gamma+\alpha)}\left|\left(\frac{\epsilon_{1}^{\rho}-t_{k}^{\rho}}{\rho}\right)^{\alpha}-\left(\frac{\epsilon_{2}^{\rho}-t_{k}^{\rho}}{\rho}\right)^{\alpha}\right| .
\end{aligned}
$$

Note that

$$
\left|\left(\frac{\epsilon_{1}^{\rho}-t_{k}^{\rho}}{\rho}\right)^{1-\gamma}\left(Q_{2} z\right)\left(\epsilon_{1}\right)-\left(\frac{\epsilon_{2}^{\rho}-t_{k}^{\rho}}{\rho}\right)^{1-\gamma}\left(Q_{2} z\right)\left(\epsilon_{2}\right)\right| \rightarrow 0 \text { as } \epsilon_{1} \rightarrow \epsilon_{2} .
$$

This shows that $Q_{2} B_{\eta}$ is equicontinuous. Therefore, $Q_{2} B_{\eta}$ is relatively compact. By $P C_{\gamma}$-type Arzela-Ascoli theorem, $Q_{2}$ is compact. As a consequence of Theorem 16, we deduce that $\Psi$ has at least a fixed point $u^{*} \in P C_{\gamma, \rho}(J)$ and by the same way of the proof of Theorem 22, we can easily show that $u^{*} \in P$ $C_{\gamma, \rho}(J)$. Using Lemma 21, we conclude that problems $((2))-((4))$ have at least one solution in the space $P C_{\gamma, \rho}^{\gamma}(J)$.

\section{Ulam-Hyers-Rassias Stability}

Now, we are concerned with the Ulam-Hyers-Rassias stability of our problems (2)-(4).

Theorem 25. Assume that in addition to (H1)-(H3) and (61), the following hypothesis holds: 
(H6). There exist a nondecreasing function $\vartheta \in P C_{\gamma, \rho}(J)$ and $\lambda_{\vartheta}, \tilde{\lambda}_{\vartheta}>0$ such that for each $t \in(a, b]$, we have

$$
\left({ }^{\rho} \mathscr{J}_{a^{+}}^{\alpha} \vartheta\right)(t) \leq \lambda_{\vartheta} \vartheta(t .)
$$

and

$$
\left(\rho \mathcal{F}_{a^{+}}^{1-\gamma} \vartheta\right)(t) \leq \tilde{\lambda}_{\vartheta} \vartheta(t .)
$$
$\vartheta, \tau)$

Then, Equation (2) is U-H-R stable with respect to

Proof. Consider the operator $\Psi$ defined in (53). Let $u \in P$ $C_{\gamma, \rho}(J)$ be a solution of inequality (27), and let us assume that $w$ is the unique solution of the problem

$$
\left\{\begin{array}{l}
\left({ }^{\rho} D_{t_{k}^{+}}^{\alpha, \beta} w\right)(t)=f\left(t, w(t),\left({ }^{\rho} D_{t_{k}^{+}}^{\alpha, \beta} w\right)(t) ; t \in J_{k}, k=0, \cdots, m,\right) \\
\left({ }^{\rho} \mathcal{g}_{t_{k}^{+}}^{1-\gamma} w\right)\left(t_{k}^{+}\right)=\left(\rho \mathcal{F}_{t_{k-1}^{+}}^{1-\gamma} w\right)\left(t_{k}^{-}\right)+L_{k}\left(w\left(t_{i}^{-}\right)\right), k=1, \cdots, m, \\
c_{1}\left({ }^{\rho} \mathcal{F}_{a^{+}}^{1-\gamma} w\right)\left(a^{+}\right)+c_{2}\left(\rho^{\rho} \mathcal{F}_{t_{m}^{+}}^{1-\gamma} w\right)(b)=c_{3}, \\
\left(\rho \mathcal{F}_{a^{+}}^{1-\gamma} w\right)\left(a^{+}\right)=\left(\rho \mathcal{F}_{a^{+}}^{1-\gamma} u\right)\left(a^{+}\right) .
\end{array}\right.
$$

By Lemma 21, we obtain for each $t \in(a, b]$

$$
\begin{aligned}
w(t)= & \frac{1}{\Gamma(\gamma)}\left(\frac{t^{\rho}-t_{k}^{\rho}}{\rho}\right)^{\gamma-1}\left[\left(\rho \mathcal{F}_{a^{+}}^{1-\gamma} w\right)\left(a^{+}\right)+\sum_{a<t_{k}<t} L_{k}\left(w\left(t_{i}^{-}\right)\right)\right. \\
& \left.+\sum_{a<t_{k}<t}\left(\rho \mathcal{F}_{\left(t_{i-1}\right)^{+}}^{1-\gamma+\alpha} h\right)\left(t_{k}\right)\right]+\left(\rho \mathcal{F}_{t_{k}^{+}}^{\alpha} h\right)(t), t \in J_{k}, k=0, \cdots, m,
\end{aligned}
$$

where $h:(a, b] \rightarrow \mathbb{R}$ is a function satisfying the functional equation

$$
h(t)=f(t, w(t), h(t)) .
$$

Since $u$ is a solution of inequality (27), by Remark 2.18, we have

$$
\left\{\begin{array}{l}
\left({ }^{\rho} D_{t_{k}^{+}}^{\alpha, \beta} u\right)(t)=f\left(t, u(t),\left({ }^{\rho} D_{t_{k}^{+}}^{\alpha, \beta} u\right)(t)\right)+\sigma(t), t \in J_{k}, k=0, \cdots, m, \\
\left({ }^{\rho} \mathcal{F}_{t_{k}^{+}}^{1-\gamma}\right)\left(t_{k}^{+}\right)=\left(\rho^{\rho} \mathcal{G}_{t_{k-1}^{+}}^{1-\gamma} u\right)\left(t_{k}^{-}\right)+L_{k}\left(u\left(t_{i}^{-}\right)\right)+\sigma_{k}, k=1, \cdots, m .
\end{array}\right.
$$

Clearly, the solution of (115) is given by

$$
\begin{aligned}
u(t)= & \frac{1}{\Gamma(\gamma)}\left(\frac{t^{\rho}-t_{k}^{\rho}}{\rho}\right)^{\gamma-1}\left[\left(\rho \mathcal{F}_{a^{+}}^{1-\gamma} u\right)\left(a^{+}\right)+\sum_{a<t_{k}<t} L_{k}\left(u\left(t_{i}^{-}\right)\right)\right. \\
& +\sum_{a<t_{k}<t} \sigma_{k}+\sum_{a<t_{k}<t}\left(\rho \mathcal{F}_{\left(t_{i-1}\right)^{+}}^{1-\gamma+\alpha} g\right)\left(t_{k}\right) \\
& \left.+\sum_{a<t_{k}<t}\left(\rho \mathcal{F}_{\left(t_{i-1}\right)}^{1-\gamma+\alpha} \sigma\right)\left(t_{k}\right)\right]+\left(\rho \mathcal{F}_{t_{k}}^{\alpha} g\right)(t) \\
& +\left(\rho \mathcal{F}_{t_{k}}^{\alpha} \sigma\right)(t), \quad t \in J_{k}, k=0, \cdots, m,
\end{aligned}
$$

where $g:(a, b] \rightarrow \mathbb{R}$ is a function satisfying the functional equation

$$
g(t)=f(t, u(t), g(t)) .
$$

Hence, for each $t \in(a, b]$, we have

$$
\begin{aligned}
|u(t)-w(t)| \leq & \frac{1}{\Gamma(\gamma)}\left(\frac{t^{\rho}-t_{k}^{\rho}}{\rho}\right)^{\gamma-1}\left[\sum_{k=1}^{m}\left|L_{k}\left(u\left(t_{k}^{-}\right)\right)-L_{k}\left(w\left(t_{k}^{-}\right)\right)\right|\right. \\
& +\sum_{k=1}^{m}\left|\sigma_{k}\right|+\sum_{k=1}^{m}\left(\rho \mathcal{F}_{\left(t_{i-1}\right)^{+}}^{1-\gamma+\alpha}|g(s)-h(s)|\right)\left(t_{k}\right) \\
& \left.+\sum_{k=1}^{m}\left(\rho \mathcal{F}_{\left(t_{i-1}\right)^{+}}^{1-\gamma+\alpha}|\sigma(s)|\right)\left(t_{k}\right)\right] \\
& +\left(\rho \mathcal{F}_{t_{k}^{+}}^{\alpha}|\mathcal{g}(s)-h(s)|\right)(t)+\left(\rho \mathcal{F}_{t_{k}^{+}}^{\alpha}|\sigma(s)|\right)(t) .
\end{aligned}
$$

Thus,

$$
\begin{aligned}
\|u-w\|_{P C_{\gamma, \rho}} \leq & \frac{1}{\Gamma(\gamma)}\left[m \epsilon \tau+\left(m \tilde{\lambda}_{\vartheta}+1\right) \epsilon \lambda_{\vartheta} \vartheta(t)\right. \\
& +\sum_{k=1}^{m} l^{*}\left|u\left(t_{i}^{-}\right)-w\left(t_{i}^{-}\right)\right| \\
& \left.+\sum_{k=1}^{m}\left(\rho \mathcal{g}_{\left(t_{k-1}\right)^{+}-\gamma+\alpha}|g(s)-h(s)|\right)\left(t_{k}\right)\right] \\
& +\left(\frac{t^{\rho}-t_{k}^{\rho}}{\rho}\right)^{1-\gamma}\left(\rho \mathcal{F}_{t_{k}^{+}}^{\alpha}|g(s)-h(s)|\right)(t) .
\end{aligned}
$$

By (H2) and Lemma 6, for $t \in(a, b]$, we have

$$
\begin{aligned}
\|u-w\|_{P C_{\gamma, \rho}} \leq & \frac{1}{\Gamma(\gamma)}\left[m \epsilon \tau+\left(m \tilde{\lambda}_{\vartheta}+1\right) \epsilon \lambda_{\vartheta} \vartheta(t)+m l^{*} p^{*}\right. \\
& \left.\cdot\|u-w\|_{P C_{\gamma, \rho}}\right]+\left[\frac{m K}{(1-M) \Gamma(1+\alpha)}\left(\frac{t_{k}^{\rho}-t_{k-1}^{\rho}}{\rho}\right)^{\alpha}\right. \\
& \left.+\frac{K \Gamma(\gamma)}{(1-M) \Gamma(\gamma+\alpha)}\left(\frac{t^{\rho}-t_{k}^{\rho}}{\rho}\right)^{\alpha}\right]\|u-w\|_{P C_{\gamma, \rho}}
\end{aligned}
$$


Thus,

$$
\begin{aligned}
\|u-w\|_{P C_{\gamma, \rho} \leq} & \frac{1}{\Gamma(\gamma)}\left(m \epsilon \tau+\left(m \tilde{\lambda}_{\vartheta}+1\right) \epsilon \lambda_{\vartheta} \vartheta(t)\right) \\
& +\left[\frac{m l^{*} p^{*}}{\Gamma(\gamma)}+\frac{K}{1-M}\left(\frac{m}{\Gamma(1+\alpha)}+\frac{\Gamma(\gamma)}{\Gamma(\lambda+\alpha)}\right)\right. \\
& \left.\cdot\left(\frac{b^{\rho}-a^{\rho}}{\rho}\right)^{\alpha}\right]\|u-w\|_{P C_{\gamma, \rho}} .
\end{aligned}
$$

Then, by (61), we have

$$
\|u-w\|_{P C_{\gamma, \rho}} \leq a_{\vartheta} \epsilon(\tau+\vartheta(t)),
$$

where

$$
\begin{aligned}
a_{\vartheta}= & \frac{1}{\Gamma(\gamma)}\left(m+\left(m \tilde{\lambda}_{\vartheta}+1\right) \lambda_{0}\right) \\
& \cdot\left[1-\frac{m l^{*} p^{*}}{\Gamma(\gamma)}+\frac{K}{1-M}\left(\frac{m}{\Gamma(1+\alpha)}+\frac{\Gamma(\gamma)}{\Gamma(\gamma+\alpha)}\right)\left(\frac{b^{\rho}-a^{\rho}}{\rho}\right)^{\alpha}\right]^{-1} .
\end{aligned}
$$

Hence, Equation (2) is U-H-R stable with respect to $(\vartheta, \tau)$.

\section{Examples}

Example 1. Consider the following impulsive BVP of generalized Hilfer Fractional differential equation

$$
\begin{aligned}
&\left({ }^{1 / 2} D_{t_{k}^{+}}^{1 / 2,0} u\right)(t)= \frac{1}{97 e^{t+2}\left(1+|u(t)|+\left|1 / 2 D_{t_{k}^{+}}^{1 / 2,0} u(t)\right|\right.} \\
&+\frac{\ln (e+\sqrt{t})}{e^{2} \sqrt{t-1}}, \text { for each } t \in J_{0} \cup J_{1}, \\
&\left({ }^{1 / 2} \mathscr{F}_{e^{+}}^{1 / 2} u\right)\left(e^{+}\right)-\left({ }^{1 / 2} \mathscr{J}_{1^{+}}^{1 / 2} u\right)\left(e^{-}\right)=\frac{\left|u\left(e^{-}\right)\right|}{3+\left|u\left(e^{-}\right)\right|}, \\
& 3\left({ }^{1 / 2} \mathscr{F}_{1^{+}}^{1 / 2} u\right)\left(1^{+}\right)-2\left({ }^{1 / 2} \mathscr{F}_{e^{+}}^{1 / 2} u\right)(3)=0,
\end{aligned}
$$

where $J_{0}=(1, e], J_{1}=(e, 3], t_{0}=1$, and $t_{1}=e$.

Set

$f(t, u, w)=\frac{1}{97 e^{t+2}(1+|u|+|w|)}+\frac{\ln (e+\sqrt{t})}{e^{2} \sqrt{t-1}}, t \in(1,3], u, w \in \mathbb{R}$.

We have

$$
\begin{aligned}
P C_{\gamma, \rho}^{\beta(1-\alpha)}([1,3]) & =P C_{1 / 2,1 / 2}^{0}([1,3]) \\
& =\left\{g:(1,3] \rightarrow \mathbb{R}: \sqrt{2}\left(\sqrt{t-t_{k}}\right)^{1 / 2} g \in P C([1,3])\right\},
\end{aligned}
$$

with $\gamma=\alpha=1 / 2, \rho=1 / 2, \beta=0$, and $k \in\{0,1\}$. Clearly, the continuous function $f \in P C_{1 / 2,1 / 2}^{0}([1,3])$.

Hence, the condition (H1) is satisfied.

For each $u, \bar{u}, w, \bar{w}, \in \mathbb{R}$ and $t \in(1,3]$,

$$
\begin{aligned}
|f(t, u, w)-f(t, \bar{u}, \bar{w})| & \leq \frac{1}{97 e^{t+2}}(|u-\bar{u}|+|w-\bar{w}|) \\
& \leq \frac{1}{97 e^{3}}(|u-\bar{u}|+|w-\bar{w}|) .
\end{aligned}
$$

Hence, condition (H2) is satisfied with $K=M=1 / 97 e^{3}$. And let

$$
L_{1}(u)=\frac{u}{3+u}, u \in[0, \infty)
$$

Let $\mathrm{u}, \mathrm{w} \in[0, \infty)$. Then, we have

$$
\left|L_{1}(u)-L_{1}(w)\right|=\left|\frac{u}{3+u}-\frac{w}{3+w}\right|=\frac{3|u-w|}{(3+u)(3+w)} \leq \frac{1}{3}|u-w|,
$$

and so the condition $(\mathrm{H} 3)$ is satisfied and $l *=1 / 3$.

A simple computation shows that condition (61) of Theorem 22 is satisfied, for

$$
\begin{aligned}
L= & \frac{1}{\sqrt{2 \pi(\sqrt{e-1})}}+\frac{3 \sqrt{2}(\sqrt{3}-1)^{1 / 2}}{\left(97 e^{3}-1\right) \Gamma(3 / 2)}+\frac{\sqrt{2}(\sqrt{3}-1)^{1 / 2}}{\left(97 e^{3}-1\right)} \\
& \cdot\left(\frac{2}{\Gamma(3 / 2)}+\sqrt{\pi}\right) \approx 0.52720987569<1 .
\end{aligned}
$$

Then, problems $((124))-((126))$ have a unique solution in $P C_{1 / 2,1 / 2}^{1 / 2}([1,3]) \subset P C_{1 / 2,1 / 2}^{1 / 2,0}([1,3])$.

Also, hypothesis (H6) is satisfied with $\vartheta(t)=e^{5}, \tau=1$, and $\lambda_{\vartheta}=\tilde{\lambda}_{\vartheta}=2 /(\Gamma(3 / 2))$. Indeed, for each $t \in J_{0} \cup J_{1}$, we get

$$
\left(\rho \mathcal{F}_{1^{+}}^{1 / 2} \vartheta\right)(t) \leq \frac{2 e^{5}}{\Gamma(3 / 2)}=\lambda_{\vartheta} \vartheta(t)=\tilde{\lambda}_{\vartheta} \vartheta(t) .
$$

Consequently, Theorem 25 implies that Equation (124) is U-H-R stable.

Example 2. Consider the following impulsive initial value problem of generalized Hilfer fractional differential equation:

$$
\begin{aligned}
& \left({ }^{1} D_{t_{k}^{+}}^{1 / 2,0} u\right)(t) \\
& \quad=\frac{3+|u(t)|+\left|{ }^{1} D_{t_{k}^{+}}^{1 / 2,0} u(t)\right|}{53 e^{-t+4}\left(1+|u(t)|+\left|{ }^{1} D_{t_{k}^{+}}^{1 / 2,0} u(t)\right|\right)}, \text { for each } t \in J_{0} \cup J_{1},
\end{aligned}
$$




$$
\begin{aligned}
\left({ }^{1} \mathscr{F}_{e^{+}}^{1 / 2} u\right)\left(e^{+}\right)-\left({ }^{1} \mathscr{F}_{1^{+}}^{1 / 2} u\right)\left(e^{-}\right) & =\frac{\left|u\left(e^{-}\right)\right|}{2+u\left(e^{-}\right)}, \\
\left({ }^{1} \mathscr{F}_{1^{+}}^{1 / 2} u\right)\left(1^{+}\right) & =0,
\end{aligned}
$$

where $J_{0}=(1, e], J_{1}=(e, 3], t_{0}=1$, and $t_{1}=e$.

Set

$$
f(t, u, w)=\frac{3+|u|+|w|}{53 e^{-t+4}(1+|u|+|w|)}, t \in(1,3], u, w \in \mathbb{R} .
$$

We have

$$
\begin{aligned}
P C_{\gamma, \rho}^{\beta(1-\alpha)}([1,3]) & =P C_{1 / 2,1}^{0}([1,3]) \\
& =\left\{g:(1,3] \rightarrow \mathbb{R}:\left(\sqrt{t-t_{k}}\right) g \in P C([1,3])\right\},
\end{aligned}
$$

with $\gamma=\alpha=1 / 2, \rho=1, \beta=0$, and $k \in\{0,1\}$. Clearly, the continuous function $f \in P C_{1 / 2,1}^{0}([1,3])$.

Hence, the condition (H1) is satisfied. For each $u, w \in \mathbb{R}$ and $\in(1,3]$,

$$
|f(t, u, w)| \leq \frac{1}{53 e^{-t+4}}(3+|u|+|w|) .
$$

Hence, condition (H4) is satisfied with $p_{1}(t)=3 / 53 e^{-t+4}$, $p_{2}(t)=p_{3}(t)=3 / 53 e^{-t+4}, p_{1}^{*}=3 / 53 e$, and $p_{2}^{*}=p_{3}^{*}=1 / 53 e$.

And let

$$
L_{1}(u)=\frac{u}{2+u}, u \in[0, \infty) .
$$

Let $u \in[0, \infty)$. Then, we have

$$
\left|L_{1}(u)\right| \leq \frac{1}{2}|u|+2,
$$

and so the condition (H5) is satisfied with $\Phi_{1}=1 / 2$ and $\Phi_{2}=2$.

The condition (74) of Theorem 23 is satisfied, for

$$
\begin{aligned}
\left(\left|\xi_{1}\right|\right. & +1)\left(\frac{m \Phi_{1} p^{*}}{\Gamma(\gamma)}+\frac{m p_{2}^{*}\left(b^{\rho}-a^{\rho}\right)^{\alpha}}{\left(1-p_{3}^{*}\right) \Gamma(1+\alpha) \rho^{\alpha}}\right) \\
& +\left(\frac{\left|\xi_{1}\right|}{\Gamma(1+\alpha)}+\frac{\Gamma(\gamma)}{\Gamma(\gamma+\alpha)}\right)\left(\frac{p_{2}^{*}\left(b^{\rho}-a^{\rho}\right)^{\alpha}}{\left(1-p_{3}^{*}\right) \rho^{\alpha}}\right) \\
= & \left(\frac{1}{2 \sqrt{2 \pi}}+\frac{\sqrt{2}}{(53 e-1) \Gamma(3 / 2)}\right)+\frac{\sqrt{2 \pi}}{53 e-1} \\
\approx & 0.22814541069 \leq 1 .
\end{aligned}
$$

Then, problems ((134)) $-((136))$ have at least one solution in $P C_{1 / 2,1}^{1 / 2}([1,3]) \subset P C_{1 / 2,1}^{1 / 2,0}([1,3])$. Also, hypothesis $(\mathrm{H} 6)$ is sat- isfied with $\vartheta(t)=t-1, \tau=1$, and $\lambda_{\vartheta}=\tilde{\lambda}_{9}=\sqrt{2} \Gamma(2) / \Gamma(5 / 2)$. Indeed, for each $t \in J_{0} \cup J_{1}$, we get

$$
\left(\rho \mathcal{F}_{1^{+}}^{1 / 2} \vartheta\right)(t) \leq \frac{\sqrt{2} \Gamma(2)}{\Gamma(5 / 2)}(t-1)=\lambda_{\vartheta} \vartheta(t)=\tilde{\lambda}_{\vartheta} \vartheta(t) .
$$

Consequently, by a simple change of the constants $l^{*}, K$, and $M$ from (H1) and (H2) to $\Phi_{1}, p_{2}^{*}$, and $p_{3}^{*}$ from (H4) and (H5), Theorem 25 implies that Equation (134) is G.U-H-R stable.

Example 3. Consider the following impulsive antiperiodic boundary value problem of generalized Hilfer Fractional differential equation:

$$
\begin{aligned}
& \left({ }^{\rho} D_{t_{k}^{+}}^{1 / 2,0} u\right)(t) \\
& \quad=\frac{e^{2}+|u(t)|+\left|{ }^{1} D_{t_{k}^{+}}^{1 / 2,0} u(t)\right|}{77 e^{-t+2}\left(1+|u(t)|+\left|{ }^{1} D_{t_{k}^{+}}^{1 / 2,0} u(t)\right|\right)}, \text { for each } t \in J_{k}, k=0, \cdots, 4,
\end{aligned}
$$

$$
\left({ }^{1} \mathcal{F}_{t_{k}^{+}}^{1 / 2} u\right)\left(t_{k}^{+}\right)-\left({ }^{\rho} \mathcal{F}_{t_{(k-1)}^{+}}^{1 / 2} u\right)\left(t_{i}^{-}\right)=\frac{\left|u\left(t_{k}^{-}\right)\right|}{10 k+\left|u\left(t_{k}^{-}\right)\right|}, k=1, \cdots, 4,
$$

$$
\left({ }^{1} \mathscr{F}_{1^{+}}^{1 / 2} u\right)\left(1^{+}\right)=-\left({ }^{1} \mathscr{F}_{9 / 5^{+}}^{1 / 2} u\right)(2)
$$

where $J_{k}=\left(t_{k}, t_{k+1}\right], t_{k}=1+(k / 5)$, for $k=0, \cdots, 4, m=4$, $a=t_{0}=1$, and $b=t_{5}=2$.

Set

$$
f(t, u, w)=\frac{e^{2}+|u|+|w|}{77 e^{-t+2}(1+|u|+|w|)}, t \in(1,2], u, w \in \mathbb{R}
$$

We have

$$
\begin{aligned}
P C_{\gamma, \rho}^{\beta(1-\alpha)}([1,2]) & =P C_{1 / 2,1}^{0}([1,2]) \\
& =\left\{g:(1,2] \rightarrow \mathbb{R}:\left(\sqrt{t-t_{k}}\right) g \in C([1,2])\right\},
\end{aligned}
$$

with $\gamma=\alpha=1 / 2, \rho=1, \beta=0$, and $k=0, \cdots, 4$. Clearly, the continuous function $f \in P C_{1 / 2,1}^{0}([1,2])$.

Hence, the condition (H1) is satisfied. For each $u, w \in \mathbb{R}$ and $\mathrm{t} \in(1,2]$,

$$
|f(t, u, w)| \leq \frac{1}{77 e^{-t+2}}\left(e^{2}+|u|+|w|\right) .
$$

Hence, condition (H4) is satisfied with $p_{1}(t)=e^{2} / 77 e^{-t+2}$, $p_{2}(t)=p_{3}(t)=1 / 77 e^{-t+2}, p_{1}^{*}=e^{2} / 77$, and $p_{2}^{*}=p_{3}^{*}=1 / 77$. 
And let

$$
L_{k}(u)=\frac{u}{10 k+u}, k=1, \cdots, 4, u \in[0, \infty) .
$$

Let $u \in[0, \infty)$. Then, we have

$$
L_{k}(u)=\frac{1}{10}|u|+1, \quad k=1, \cdots, 4,
$$

and so the condition (H5) is satisfied with $\Phi_{1}=1 / 10$ and $\Phi_{2}=1$.

The condition (90) of Theorem 24 is satisfied, for

$$
\begin{aligned}
\left(\left|\xi_{1}\right|+1\right) & \left(\frac{m \Phi_{1} p^{*}}{\Gamma(\gamma)}+\frac{m p_{2}^{*}\left(b^{\rho}-a^{\rho}\right)^{\alpha}}{\left(1-p_{3}^{*}\right) \Gamma(1+\alpha) \rho^{\alpha}}\right) \\
& +\frac{p_{2}^{*}\left|\xi_{1}\right|\left(b^{\rho}-a^{\rho}\right)^{\alpha}}{\left(1-p_{3}^{*}\right) \Gamma(1+\alpha) \rho^{\alpha}}=\frac{3 \sqrt{5}}{5 \sqrt{\pi}}+\frac{125}{1463 \Gamma(3 / 2)}<1 .
\end{aligned}
$$

Then, problems ((144)) $-((146))$ have at least one solution in $P C_{1 / 2,1}^{1 / 2}([1,2]) \subset P C_{1 / 2,1}^{1 / 2,0}([1,2])$. Also, hypothesis (H6) is satisfied with $\vartheta(t)=(1-t)^{2}, \tau=1$, and $\lambda_{\vartheta}=\tilde{\lambda}_{\vartheta}=\Gamma(3) / \Gamma(7 / 2)$. Indeed, for each $t \in J_{k}, k=0, \cdots, 4$, we get

$$
\left(\rho \mathcal{F}_{1^{+}}^{1 / 2} \vartheta\right)(t) \leq \frac{\Gamma(3)}{\Gamma(7 / 2)}(t-1)^{2}=\lambda_{\vartheta} \vartheta(t)=\tilde{\lambda}_{\vartheta} \vartheta(t)
$$

The same as Example 2, Theorem 25 implies that Equation (144) is U-H-R stable.

\section{Conclusion}

In this paper, we are concerned with existence, uniqueness, and Ulam-Hyers-Rassias stability results for a class of boundary value problem for nonlinear implicit fractional differential equations with impulses and generalized Hilfer fractional derivative. We gave three results using the Banach contraction principle and Krasnoselskii's and Schaefer's fixed-point theorems. Two illustrative examples about the uniqueness and the stability were presented. An interesting extension of our research would be to address UlamHyers-Mittag-Leffler stability for a class and coupled system of implicit fractional differential equations. This subject will be discussed in the forthcoming papers.

\section{Data Availability}

There is no data used during this research.

\section{Conflicts of Interest}

The authors declare that they have no conflicts of interest.

\section{References}

[1] S. Abbas, M. Benchohra, J. R. Graef, and J. Henderson, Impli cit Fractional Differential and Integral Equations: Existence and Stability, De Gruyter, London, 2018.
[2] S. Abbas, M. Benchohra, and G. M. N'Guérékata, Topics in Fractional Differential Equations, vol. 27 of Developments in Mathematics, Springer New York, New York, 2012.

[3] S. Abbas, M. Benchohra, and G. M. N'Guerekata, Advanced Fractional Differential and Integral Equations, Nova Science Publishers, New York, 2014.

[4] B. Ahmad, A. Alsaedi, S. K. Ntouyas, and J. Tariboon, Hadamard-Type Fractional Differential Equations, Inclusions and Inequalities, Springer, Switzerland, 2017.

[5] B. Ahmad and S. K. Ntouyas, "Fractional differential inclusions with fractional separated boundary conditions," Fractional Calculus and Applied Analysis, vol. 15, no. 3, pp. 362$382,2012$.

[6] D. Baleanu, New Trends in Nanotechnology and Fractional Calculus Applications, Springer, Dordrecht, 2010.

[7] M. Benchohra, S. Bouriah, and J. R. Graef, "Boundary value problems for nonlinear implicit Caputo-Hadamard-type fractional differential equations with impulses," Mediterranean Journal of Mathematics, vol. 14, no. 5, p. 206, 2017.

[8] M. Benchohra, J. Henderson, and S. K. Ntouyas, Impulsive Differential Equations and Inclusions, vol. 2, Hindawi Publishing Corporation, New York, 2006.

[9] M. Benchohra and J. E. Lazreg, "Existence results for nonlinear implicit fractional differential equations with impulse," Communications in Applied Analysis, vol. 19, pp. 413-426, 2015.

[10] A. Salim, M. Benchohra, E. Karapinar, and J. E. Lazreg, "Existence and Ulam stability for impulsive generalized Hilfer-type fractional differential equations," Advances in Difference Equations, vol. 2020, no. 1, Article ID 3063, 21 pages, 2020.

[11] A. Salim, M. Benchohra, J. E. Lazreg, and J. Henderson, "Nonlinear implicit generalized Hilfer-type fractional differential equations with non-instantaneous impulses in Banach spaces," Advances in the Theory of Nonlinear Analysis and its Application, vol. 4, no. 4, pp. 332-348, 2020.

[12] A. Salim, M. Benchohra, J. R. Graef, and J. E. Lazreg, "Boundary value problem for fractional generalised Hilfer-type fractional derivative with non-instantaneous impulses," Fractal and Fractional, vol. 5, no. 1, pp. 1-21, 2021.

[13] A. M. AHMAD and N. E. TATAR, "Nonexistence of global solutions for a fractional system of strongly coupledintegrodifferential equations," Turkish Journal of Mathematics, vol. 43, no. 6, pp. 2715-2730, 2019.

[14] M. D. Kassim and N. Tatar, "Convergence of solutions of fractional differential equations to power-type functions," Electronic Journal of Differential Equations, 2020.

[15] C. Li and S. Sarwar, "Linearization of nonlinear fractional differential systems with Riemann-Liouville and Hadamard derivatives," Progress in Fractional Differentiation and Applications, vol. 6, pp. 11-22, 2020.

[16] C. Li and S. Sarwar, "Existence and continuation of solutions for Caputo type fractional differential equations," Electronic Journal of Differential Equations, vol. 207, pp. 1-14, 2016.

[17] A. Seemab, M. Ur Rehman, J. Alzabut, and A. Hamdi, "On the existence of positive solutions for generalized fractional boundary value problems," Boundary Value Problems, vol. 2019, no. 1, Article ID 186, 2019.

[18] J. V. C. Sousa, F. Jarad, and T. Abdeljawad, "Existence of mild solutions to Hilfer fractional evolution equations in Banach space," Annals of Functional Analysis, vol. 12, no. 1, pp. 116, 2021. 
[19] R. Subashini and C. Ravichandran, "On the results of nonlocal Hilfer fractional semilinear differential inclusions," Proceedings of the Jangjeon Mathematical Society, vol. 22, pp. 249267, 2019.

[20] A. Zada, J. Alzabut, H. Waheed, and I. L. Popa, "Ulam-Hyers stability of impulsive integrodifferential equations with Riemann-Liouville boundary conditions," Advances in Difference Equations, vol. 2020, no. 1, Article ID 64, 2020.

[21] A. Zada, H. Waheed, J. Alzabut, and X. Wang, "Existence and stability of impulsive coupled system of fractional integrodifferential equations," Demonstratio Mathematica, vol. 52, no. 1, pp. 296-335, 2019.

[22] S. M. Ulam, A Collection of Mathematical Problems, Interscience Publishers, New York, 1968.

[23] D. H. Hyers, "On the stability of the linear functional equation," Proceedings of the National Academy of Sciences of the United States of America, vol. 27, no. 4, pp. 222-224, 1941.

[24] T. M. Rassias, "On the stability of the linear mapping in Banach spaces," Proceedings of the American mathematical society, vol. 72, no. 2, pp. 297-300, 1978.

[25] I. A. Rus, "Ulam stability of ordinary differential equations," Studia Universitatis Babes-Bolyai, Mathematica, vol. 25, no. 4, pp. 125-133, 2009.

[26] S. Abbas, M. Benchohra, J. E. Lazreg, and Y. Zhou, "A survey on Hadamard and Hilfer fractional differential equations: analysis and stability," Chaos, Solitons and Fractals, vol. 102, pp. 47-71, 2017.

[27] S. Abbas, M. Benchohra, J. E. Lagreg, A. Alsaedi, and Y. Zhou, "Existence and Ulam stability for fractional differential equations of Hilfer-Hadamard type," Advances in Difference Equations, vol. 2017, no. 1, Article ID 1231, 2017.

[28] M. Benchohra and J. E. Lazreg, "Existence and Ulam stability for nonlinear implicit fractional differential equations with Hadamard derivative," Studia Universitatis Babeș-Bolyai Mathematica, vol. 62, no. 1, pp. 27-38, 2017.

[29] M. Benchohra and J. E. Lazreg, "On stability for nonlinear implicit fractional differential equations," Le Matematiche, vol. 70, no. 2, pp. 49-61, 2015.

[30] S. Harikrishnan, R. W. Ibrahim, and K. Kanagarajan, "Fractional Ulam-stability of fractional impulsive differential equation involving Hilfer-Katugampola fractional differential operator," Universal Journal of Mathematics and Applications, vol. 1, pp. 106-112, 2018.

[31] U. Katugampola, "New approach to a generalized fractional integral," Applied Mathematics and Computation, vol. 218, no. 3, pp. 860-865, 2011.

[32] D. S. Oliveira and E. C. de Oliveira, "Hilfer-Katugampola fractional derivatives," Computational and Applied Mathematics, vol. 37, no. 3, pp. 3672-3690, 2018.

[33] R. Almeida, A. B. Malinowska, and T. Odzijewicz, "Fractional differential equations with dependence on the Caputo-Katugampola derivative," Journal of Computational and Nonlinear Dynamics, vol. 11, no. 6, pp. 1-11, 2016.

[34] A. Granas and J. Dugundji, Fixed Point Theory, Springer New York, New York, 2003.

[35] J. Wang, M. Fec `kan, and Y. Zhou, "Ulam's type stability of impulsive ordinary differential equations," Journal of Mathematical Analysis and Applications, vol. 395, no. 1, pp. 258264, 2012. 